Portland State University

PDXScholar

Civil and Environmental Engineering Faculty

Publications and Presentations

Civil and Environmental Engineering

$2-2014$

\title{
Time-Variable Refraction of the Internal Tide at the Hawaiian Ridge
}

\author{
Edward D. Zaron \\ Portland State University, ezaron@pdx.edu \\ Gary D. Egbert \\ Oregon State University
}

Follow this and additional works at: https://pdxscholar.library.pdx.edu/cengin_fac

Part of the Civil Engineering Commons, and the Environmental Engineering Commons Let us know how access to this document benefits you.

\section{Citation Details}

Zaron, E. D., \& Egbert, G. D. (2014). Time-Variable Refraction of the Internal Tide at the Hawaiian Ridge. Journal Of Physical Oceanography, 44(2), 538-557.

This Article is brought to you for free and open access. It has been accepted for inclusion in Civil and Environmental Engineering Faculty Publications and Presentations by an authorized administrator of PDXScholar. Please contact us if we can make this document more accessible: pdxscholar@pdx.edu. 


\title{
Time-Variable Refraction of the Internal Tide at the Hawaiian Ridge
}

\author{
EDWARD D. ZARON \\ Portland State University, Portland, Oregon \\ GARY D. EGBERT \\ Oregon State University, Corvallis, Oregon
}

(Manuscript received 14 December 2012, in final form 5 September 2013)

\begin{abstract}
The interaction of the dominant semidiurnal $M_{2}$ internal tide with the large-scale subtidal flow is examined in an ocean model by propagating the tide through an ensemble of background fields in a domain centered on the Hawaiian Ridge. The background fields are taken from the Simple Ocean Data Assimilation (SODA) ocean analysis, at 2-month intervals from 1992 through 2001. Tides are computed with the Primitive Equation Z-coordinate Harmonic Analysis of Tides (PEZ-HAT) model by 14-day integrations using SODA initial conditions and $M_{2}$ tidal forcing. Variability of the tide is found to occur primarily as the result of propagation through the nonstationary background fields, rather than via generation site variability. Generation of incoherent tidal variability is mapped and shown to occur mostly in association with waves generated at French Frigate Shoals scattering near the Musicians Seamounts to the north of the ridge. The phasecoherent internal tide loses energy at a domain-average rate of $2 \mathrm{~mW} \mathrm{~m}^{-2}$ by scattering into the nonstationary tide. Because of the interference of waves from multiple generation sites, variability of the internal tide is spatially inhomogeneous and values of the scattering rate 10 times larger occur in localized areas. It is estimated that $20 \%$ of the baroclinic tidal energy flux is lost by adiabatic scattering (refraction) within $250 \mathrm{~km}$ of the ridge, a value regarded as a lower bound because of the smoothed nature of the SODA fields used in this study.
\end{abstract}

\section{Introduction}

Approximately one-third of the energy loss from the ocean surface tide occurs in the deep ocean in association with submarine topography via the conversion of the barotropic surface tide into the baroclinic internal tide (Egbert and Ray 2001; Garrett and Kunze 2007). Once the tide becomes baroclinic there are numerous processes that may cause it to dissipate, resulting in small-scale mixing and vertical buoyancy transport in the ocean (Wunsch and Ferrari 2004). Mechanisms by which the baroclinic tide can generate turbulence include scattering by small-scale topographic features (Polzin et al. 1997); parametric subharmonic instability (MacKinnon and Winters 2005); bottom boundary layer processes near sites where the bottom slope is

Corresponding author address: Edward D. Zaron, Department of Civil and Environmental Engineering, Portland State University, P.O. Box 751, Portland, OR 97207-0751.

E-mail: zaron@cecs.pdx.edu tangent to characteristic surfaces of tidal internal waves (Levine and Boyd 2006); convective instability of large-amplitude internal waves (Klymak et al. 2008; Legg and Klymak 2008); shear-driven instability in the near-surface layers of the ocean (Cole et al. 2009) or along internal wave characteristics (Muller and Bühler 2009); and interactions with the subtidal circulation, inertial waves, mesoscale, and submesoscale flow, leading to wave refraction, scattering, or other interactions that provide a route for tidal baroclinicity to enter the internal wave continuum (Rainville and Pinkel 2006b; Chavanne et al. 2010b; Zilberman et al. 2011).

This subject of this article is the refraction of the internal tide by large-scale time-variable stratification, which is examined to quantify both the rate and mechanisms of scattering of the internal tide. The approach taken is to model the generation and propagation of the dominant semidiurnal tide $M_{2}$ within an ensemble of realistic subtidal background fields. Simultaneous modeling of the tides and mesoscales is not attempted, as this 
would entail coupling to a global model to generate a realistic mean stratification, flow instability, and eddying from first principles. Instead, subtidal background fields are taken from the Simple Ocean Data Assimilation (SODA), version 1.4.2, data-assimilative ocean hindcast (Carton and Giese 2008), and the tidal fields are computed by solving an initial value problem utilizing the SODA fields augmented with tidal forcing.

Time variation of the background fields causes the phase and amplitude of the tidal solutions to vary with each realization. Ensemble-average tidal fields will be referred to as the coherent tide, because these would be the tidal signal detectable in a harmonic analysis of long time series. The difference between the coherent tide and the tide computed in a particular realization of background flow will be referred to as the incoherent tide. As the tide propagates through the ocean, there will be an apparent transfer of energy from the coherent tide to the incoherent tide caused solely by temporal variations in the background flow. The focus here is these adiabatic processes, rather than the energetic exchanges between the tides and mesoscales, or direct dissipation of the tide.

There are several motivations for this study. First, there is now a series of studies that has sought to map the baroclinic tide using satellite altimetry and to infer its energetics (Ray and Mitchum 1996; Dushaw 2002; Tian et al. 2006; Zhao and Alford 2009; Dushaw et al. 2011). But the internal tide detected by altimetry is only the component that is phase locked with the tidal forcing over the duration of the harmonic analysis, and few observations directly constrain the variability of the low-mode internal tide (Ray and Zaron 2011). The results below are a direct, if model-based, estimate of the incoherent tide, inaccessible from altimetry. Second, while good agreement between satellite observations and ocean models has been obtained for sea surface height in high-resolution regional internal tide models (e.g., Carter et al. 2008), the level of agreement is not within the expected errors of the harmonically analyzed data. To achieve improved accuracy, particularly at distance from the generation sites, it is anticipated that dataassimilative modeling will be necessary, and for this purpose tide model errors must be characterized. The approach described below has been used to provide an estimate of model error due to time variability of the mesoscale eddy field, which is neglected in present dataassimilative tide models (Zaron et al. 2009). Finally, future wide-swath satellite altimeters will measure sea surface height at length scales where the baroclinic tide is a significant, sometimes dominant, component of sea level variability. But, as with present altimeters, the orbit characteristics will alias the tides into longer periods overlapping with the time scales of ocean mesoscale and submesoscale dynamics ( $\mathrm{Fu}$ and Ferrari 2008). The present study describes the coherent and incoherent baroclinic tide, so that its contributions to future observations can be assessed.

The remainder of this paper is organized as follows. In section 2, the attributes of the tidal model and subtidal background fields are reviewed. Model sea surface height is compared with along-track satellite altimeter data in section 3, with particular emphasis on the partitioning between the coherent and incoherent tides. Baroclinic tidal energy flux is also diagnosed from the model, and it is compared with published data at several sites. The discussion section considers the role of the incoherent tide in the tidal energy budget around the Hawaiian Ridge and the possibility to identify ocean mixing caused by the decay of the internal tide.

\section{Methods}

\section{a. Tidal model}

The numerical model employed is the Primitive Equation Z-coordinate Harmonic Analysis of Tides model (abbreviated PEZ-HAT), previously used for regional tidal modeling and data assimilation (Zaron and Egbert 2007; Zaron et al. 2009; Chavanne et al. 2010a). The numerics of PEZ-HAT are based on the Geophysical Fluid Dynamics Laboratory (GFDL) Modular Ocean Model, version 3 (MOM3), modified to include partial bottom levels and a more accurate time-averaging kernel for the split-explicit barotropic mode (Zaron and Egbert $2006 \mathrm{~b})$. The model is run at $1 / 30^{\circ}(4 \mathrm{~km})$ horizontal resolution within a domain extending from $15^{\circ}$ to $32^{\circ} \mathrm{N}$ and $180^{\circ}$ to $210^{\circ} \mathrm{E}$, surrounding the Hawaiian Ridge. There are 30 levels in the vertical ranging in thickness from approximately 60 to $1500 \mathrm{~m}$, and bottom topography is subsampled from Smith and Sandwell (1997). The model domain is shown in Fig. 1 together with several sites where model and observations are compared (below).

On open boundaries the model is forced with the barotropic transport of the dominant semidiurnal $M_{2}$ tide taken from the data-assimilating barotropic tidal model of Egbert and Erofeeva (2002, version TPXO.5), which is constrained by satellite altimetry data. The $M_{2}$ component of the astronomical tide-generating force, modified to account for solid-earth loading and ocean self-attraction, is also included.

To stably integrate the three-dimensional model with these boundary conditions, the baroclinic energy generated within the model must be dissipated or permitted to leave the domain. For this purpose Rayleigh damping and increased Laplacian mixing coefficients are used in the baroclinic momentum and buoyancy conservation 


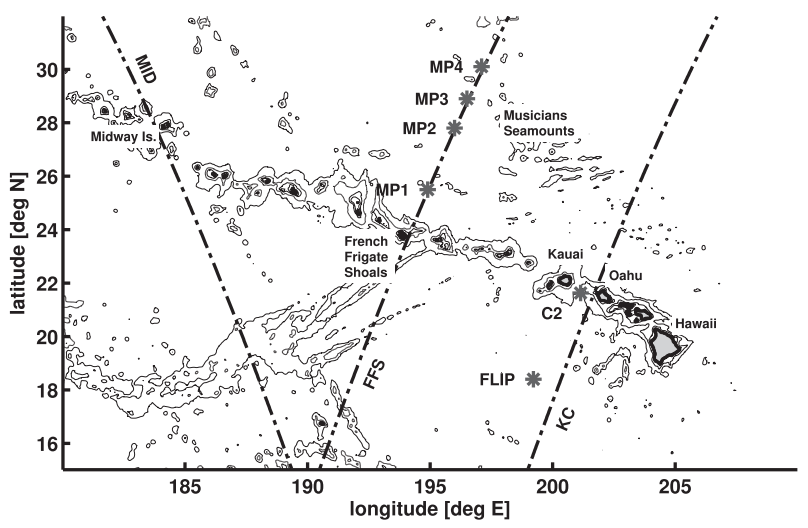

FIG. 1. The computational domain $\left(15^{\circ}-32^{\circ} \mathrm{N}, 180^{\circ}-210^{\circ} \mathrm{E}\right)$ resolved with $1 / 30^{\circ}$ resolution and 30 vertical levels. Model and observations are compared along the three TXA ground tracks passing near Midway Island, French Frigate Shoals, and Kauai Channel (MID, pass 134; FFS, pass 249; and KC, pass 223, respectively). Stations labeled MP1-MP4 are mooring sites from Alford et al. (2007). The FLIP site is the far-field location discussed in Rainville and Pinkel (2006a). The C2 site is from Zilberman et al. (2011). The 100-, 500-, 2000-, and 4000-m isobaths are contoured.

equations in sponge layers adjacent to the open boundaries, with numeric coefficients chosen to prevent the spurious reflection of baroclinic waves at the boundary (Zaron and Egbert 2006b). Radiation conditions on baroclinic velocity and buoyancy field perturbations are also used. Throughout the domain interior, Laplacian horizontal viscosity and diffusion have been reduced to the minimum level needed to prevent numerical instabilities. The lateral turbulent diffusivity is set to $20 \mathrm{~m}^{2} \mathrm{~s}^{-1}$, and the viscosity is computed via the Smagorinsky-Lilly scheme (Lilly 1967) using a $20 \mathrm{~m}^{2} \mathrm{~s}^{-1}$ minimum value. The vertical diffusivity and viscosity are set with a Richardson number-dependent scheme, which is sufficient to suppress static instabilities in the present application (Large et al. 1994).

Equivalent potential temperature is taken as the active tracer in the model. It is computed from background values of temperature, salinity, and depth to yield the correct buoyancy using a linear approximation to the equation of state (DeSzoeke 2004).

\section{b. Subtidal background fields}

The subtidal background through which the tide propagates is provided by the SODA data-assimilative hindcast model. In particular, the SODA fields are computed with a noneddy-resolving model driven by the National Centers for Environmental Prediction (NCEP) reanalysis wind stress and surface buoyancy flux. Multivariate objective analysis is used to reinitialize the SODA model at 10-day intervals using in situ and satellite observations (Carton and Giese 2008). The fields produced are thus constrained to reproduce the gross features of the quasi-steady general circulation, while also providing a coarse snapshot of the unsteady circulation. By construction, the variability in SODA is similar to what would be seen in satellite altimetry and infrared thermal imagery of the ocean surface, smoothed over a spatial scale of $300-400 \mathrm{~km}$, depending on data density (Carton et al. 2000).

Although the resolution of the SODA model is not high enough to permit vigorous baroclinic instability, a large-scale eddy field is present in the model (Fig. 2). A snapshot of the Rossby number Ro (relative vorticity divided by Coriolis parameter) and velocity vectors, both averaged over the top $100 \mathrm{~m}$, is shown in Fig. 2a. Figure $2 \mathrm{~b}$ illustrates ocean temperature and velocity fields, averaged from the surface to $500 \mathrm{~m}$. Current meanders and eddies with scales of a few hundred kilometers are present, representative of the scales of saturated baroclinic instability (Qiu 1999). At $24^{\circ} \mathrm{N}$, a time-longitude Hovmöller diagram of the mode-1 internal tide phase speed anomaly $c_{p}$ (Fig. 2c) indicates westward propagation of some anomalies close to the nominal $6 \mathrm{~cm} \mathrm{~s}^{-1}$ speed predicted for linear Rossby waves at this latitude (Chelton and Schlax 1996), although relatively few anomalies propagate unambiguously in this zonal slice. Because the particle speed exceeds the westward propagation speed of linear Rossby waves, the circulation in SODA is nonlinear in spite of being "noneddy resolving."

\section{c. Additional details}

Each member of the ensemble of tidal fields analyzed below is obtained by harmonic analysis of a PEZHAT integration utilizing SODA initial conditions from 15 January 1992 to 15 November 2000, at 2-month intervals. The full ensemble thus consists of 54 realizations of the tidal fields propagating through the large-scale eddy field. Each integration is conducted for 14 days, including a 3-day period during which the tidal forcing is ramped up. Harmonic analysis is conducted over the last 3 days of the integration. During the integration, mode- 1 internal waves in deep water can cross the entire computational domain, while mode- 2 waves can travel the distance from the ridge to the open boundary.

Note that some care is necessary when using the SODA fields for initial conditions in PEZ-HAT. In particular, the SODA velocity and buoyancy fields are relaxed to a state of no motion in the sponge layers approaching the open boundaries to avoid nonlinear instability arising from the ill posedness of the open boundary conditions (Bennett 2002). Also, the Smagorinsky-Lilly scheme used for horizontal mixing was chosen primarily as an 

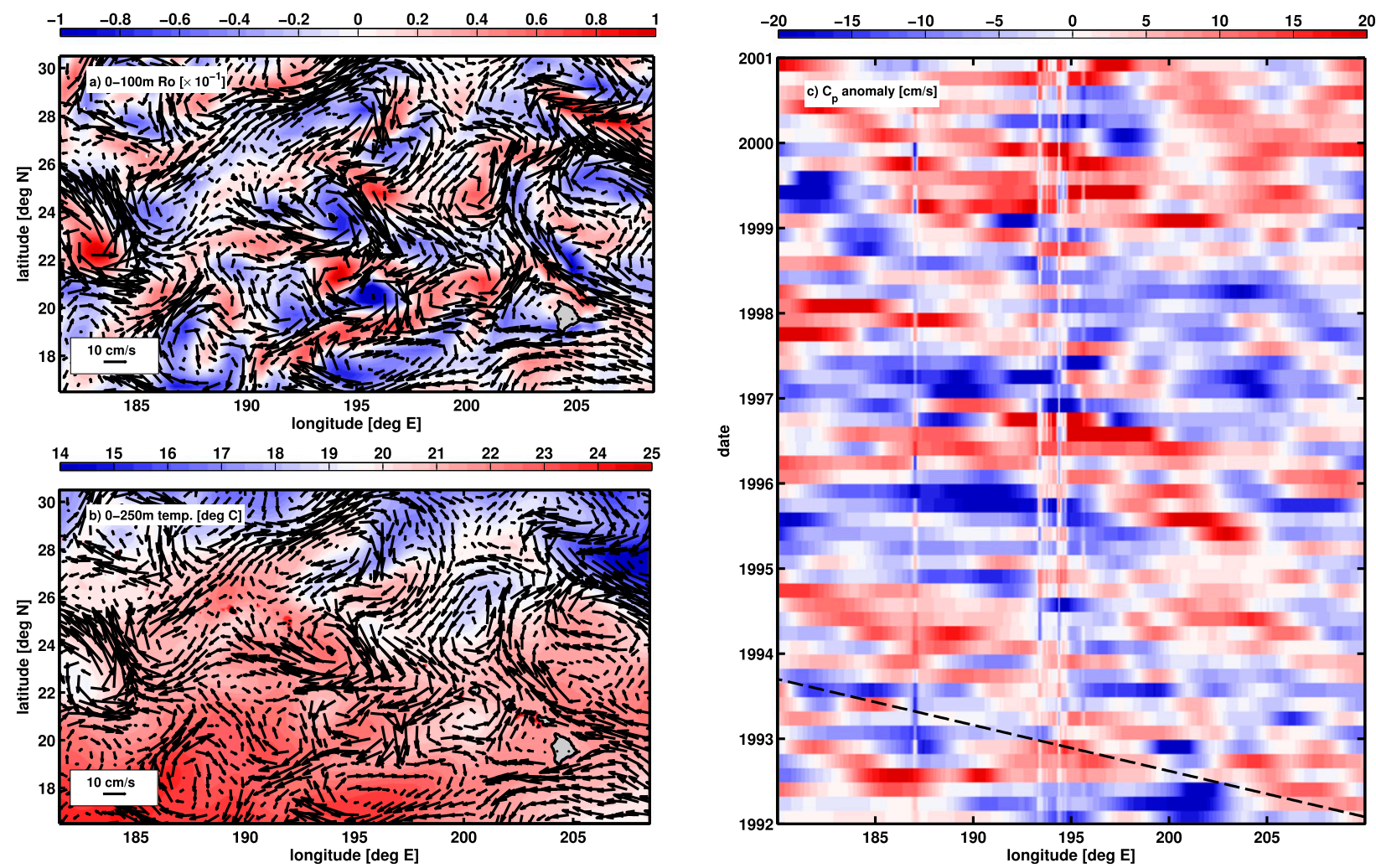

FIG. 2. Representative subtidal fields from SODA. Snapshot of March 1993 (a) Ro (color) and velocity (vectors) averaged over the top $100 \mathrm{~m}$ and (b) temperature and velocity averaged over the top $500 \mathrm{~m}$. (c) Time-lon Hovmöller plot of mode- $1 c_{p}$ anomalies at $24^{\circ} \mathrm{N}$. Dotted line corresponds to the $6 \mathrm{~cm} \mathrm{~s}^{-1}$ speed of a nondispersive mode- 1 baroclinic Rossby wave. Anomalies at fixed lon (near $187^{\circ}$ and $\left.194^{\circ} \mathrm{E}\right)$ are associated with shallow seamounts where $c_{p}$ is sensitive to near-surface density variations.

expedient to suppress the instability of initial transients created near coastlines, caused by the mismatch of the SODA and PEZ-HAT domains because of their differing resolutions. Furthermore, higher-than-linear interpolation must be used to map the SODA fields onto the PEZ-HAT grid to avoid the creation of spurious jumps in horizontal derivatives of the background fields; in the present case, the Princeton spline software library (PSPLINE) is used to compute interpolants that are twice continuously differentiable (McCune and Ludescher-Furth 2011).

Complex harmonic constants are archived for the three-dimensional potential temperature and horizontal velocity fields and the two-dimensional surface elevation field. During run time, the sea surface height and vertical velocity at the bottom are monitored for numerical instabilities. A few integrations have been examined in detail to monitor the evolution of the subtidal flow fields. During the 14-day integration, changes in the subtidal solution are generally negligible except near coastlines. Presumably processes such as baroclinic instability occur, but the time scale of the resolved instabilities is large compared to both the tidal period and the total integration time. The quasi-linear baroclinic
Rossby waves resolved by SODA propagate between 7 and $25 \mathrm{~km}$ during the 3-day harmonic analysis period, a distance that is too small to impact the resolved tides.

\section{Results}

\section{a. Sea surface height}

The internal tide is generated primarily at localized spots of strong cross-isobath tidal flow (St. Laurent and Garrett 2002). Along the Hawaiian Ridge, waves originating at different sites interfere, leading to beamlike structures in the pattern of internal wave radiation (Johnston et al. 2003; Simmons et al. 2004; Rainville et al. 2010). Using ray tracing, Rainville and Pinkel (2006b) demonstrated that the decay of the internal tide away from the Hawaiian Ridge could, in part, be explained by the loss of coherence as it propagates through the ambient mesoscale circulation. However, the ray-tracing approach of Rainville and Pinkel (2006b) is limited by the necessity to consider discrete ray paths with generation sites and directions chosen a priori. Furthermore, the geometrical optics approximation used is not formally valid because there is no scale 

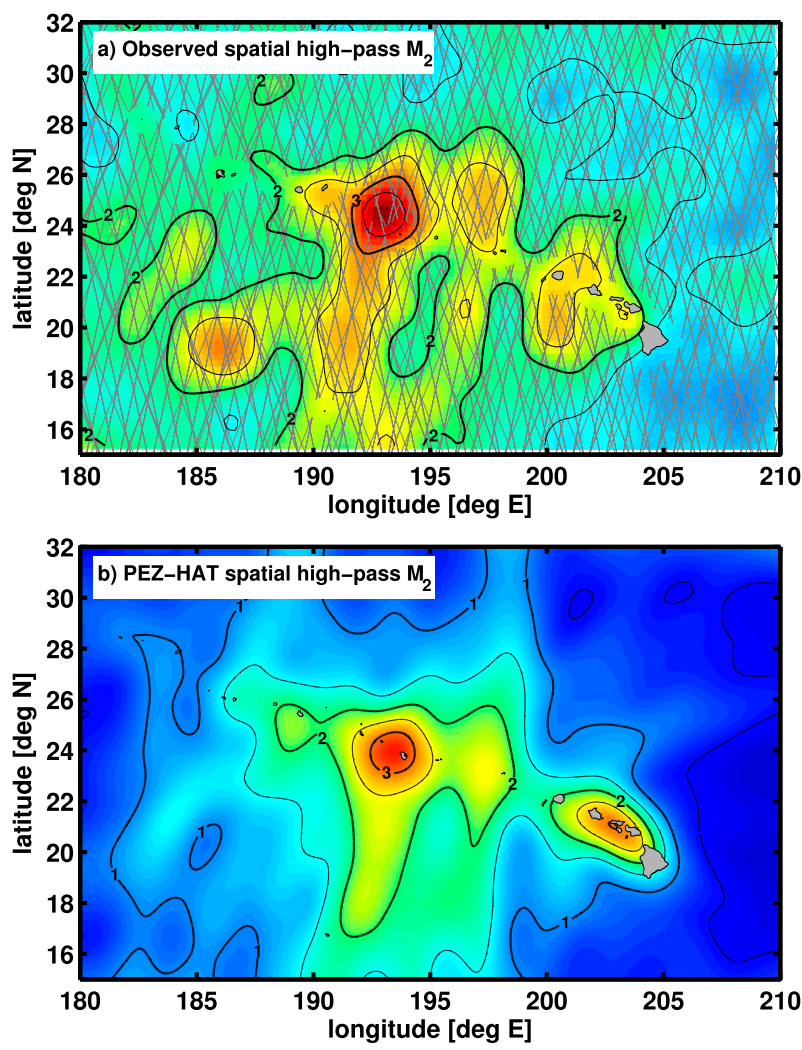

FIG. 3. Amplitude of $M_{2}$ internal tide from (a) along-track satellite altimeter data and (b) PEZ-HAT. To produce (a), the observed tide along ground tracks (gray lines) is smoothed and gridded by convolution with a $200-\mathrm{km}$ Gaussian kernel, and the amplitude of the residual high-pass-filtered fields is gridded at $1 / 4^{\circ}$ resolution. In (b), the computations are performed in two dimensions on the native model grid at $1 / 30^{\circ}$ resolution. The color scale ranges from 0 to $4 \mathrm{~cm}$, and amplitudes are contoured in $0.5 \mathrm{~cm}$ increments.

separation between the resolved eddies, 100-300 km (Qiu 1999), and the low-mode internal tide, $60-180 \mathrm{~km}$.

The $M_{2}$ internal tide generated within PEZ-HAT has been compared with the main features of the observed internal tide (Fig. 3), where the latter field has been identified by merging observations from multiple satellite altimeter missions. To create a map of the internal tide amplitude, data from Ocean Topography Experiment (TOPEX)/Poseidon (TXA), Jason-1, Jason-2, Geosat Follow-On, and the exact repeat orbit phases of Envisat-1 and ERS-2 were assembled to form time series within nominal $6-\mathrm{km}$ segments along the ground tracks of the respective missions. Standard instrumental and environmental corrections (excluding the ocean surface tide) and the CLS11 mean sea surface (Schaeffer et al. 2011) were used to convert the range data into sea surface height (SSH), avoiding sites within $60 \mathrm{~km}$ of the coast. Harmonic analysis (Foreman et al. 2009) was performed to identify the mean $Z_{0}$ and in-phase and quadrature components of the $M_{2}$ and $K_{1}$ constituents. The $M_{2}$ harmonic constants were then averaged within $1 / 4^{\circ}$ squares on a regular grid, and smoothed using a radial basis function with a $2^{\circ}$ half-power point, thus producing a smoothed and gridded map of the $M_{2}$ tide at $1 / 4^{\circ}$ resolution. The internal tide map in Fig. 3a was created by subtracting the smoothed and gridded field from the observed (along track) harmonic constants, and gridding this anomaly at $1 / 4^{\circ}$ resolution. A similar approach was used to create Fig. $3 b$, except that the spatial filtering was performed on the $1 / 30^{\circ}$ model grid.

The comparison in Fig. 3 indicates that the high-passfiltered $M_{2}$ signal is larger in the observations than in the model, but much of this difference in amplitude may be attributed to the approximate $1-\mathrm{cm}$ noise level of the observations; although, internal tides generated outside the domain would also contribute to the difference. The maps illustrate the significance of French Frigate Shoals $\left(25^{\circ} \mathrm{N}, 193^{\circ} \mathrm{E}\right)$ as the source of an $M_{2}$ internal tide beam that propagates to the southwest. Generation sites associated with Necker Island $\left(24^{\circ} \mathrm{N}\right.$, $\left.197^{\circ} \mathrm{E}\right)$ and Kauai Channel $\left(22^{\circ} \mathrm{N}, 201^{\circ} \mathrm{E}\right)$ are also evident. A notable difference between model and observations is the lack of an apparent source near $19^{\circ} \mathrm{N}, 186^{\circ} \mathrm{E}$.

Comparison of SSH in detail along the same TXA ground track segments used in Carter et al. (2008) is shown in Fig. 4. A model-data error of $1.1-1.2 \mathrm{~cm}$ is found, $1-3 \mathrm{~mm}$ larger than errors reported by Carter et al. (2008), where the identical definition of the absolute root-mean-square error has been used (Cummins and Oey 1997). Several factors could explain the difference in accuracy between the two models, but different bottom topography and resolution are the most likely explanations. The horizontal resolution of the model used in Carter et al. (2008) was approximately a factor of 4 finer than used in the present study, and the topography was derived from a high-resolution multibeam survey. In addition, the domain used in Carter et al. (2008) was comprised of roughly $1 / 30$ the area of the present model. Thus, the present results at the ridge are slightly less constrained by the open boundary conditions.

TXA data along three representative ground tracks have also been compared. Proceeding from west to east (Fig. 1), consider the track passing near MID (TXA pass 134). Figure 5 separately shows the wavenumber power spectrum of the detided (left) and $M_{2}$ tidal analysis (right) of the observed and modeled fields. On the left, one sees that the SODA SSH (solid) has a redder spectrum than observed SSH (dashed). The SODA power spectrum rolls off faster than the observed spectrum and its shape is consistent with a bell-shaped (Gaussian) 


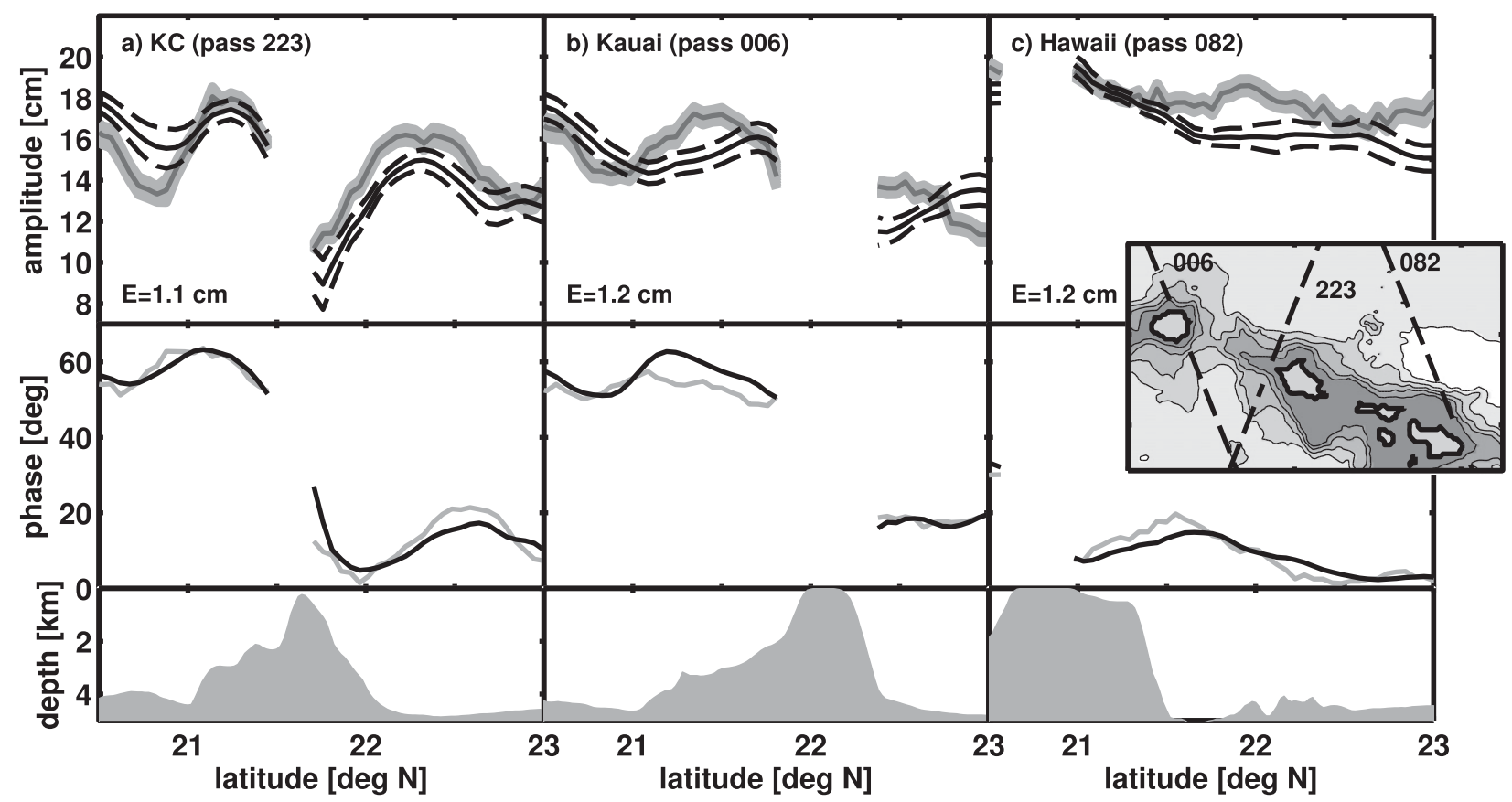

FIG. 4. Detailed comparison with altimetry near Kauai and Oahu. The $M_{2}$ surface elevation is compared with harmonically analyzed TXA data along the three ground track segments used in Carter et al. (2008). Locations of the tracks are indicated with dash lines on the inset, which also shows topography contoured at 1000-m increments. (top) Tidal amplitude observed (gray line; gray band indicates the standard error of the harmonic analysis) and modeled (black line; dash lines indicate the ensemble std dev). (middle) Greenwich phase observed (gray) and modeled (black). (bottom) Bottom topography along the ground tracks.

correlation function in physical space (Carton et al. 2000). The right shows similarity between the ensembleaveraged PEZ-HAT SSH (solid) and observed $M_{2} \mathrm{SSH}$ (dashed) spectra. Because of the orientation of the ground track, the mode- 1 signal is spread over a range of wavelengths between about 150 and $400 \mathrm{~km}$, and there is some wavenumber offset, possibly because of a misalignment of the modeled beam relative to that observed near the ground track. The incoherent tidal spectrum (red) is defined as the ensemble-average wavenumber spectrum minus the spectrum of the ensemble average. Comparing the red and black lines, one sees that SSH variance is mostly incoherent at wavelengths shorter than $150 \mathrm{~km}$.

Figure 6 compares SODA, PEZ-HAT, and observed $\mathrm{SSH}$ along the ground track that crosses French Frigate Shoals. As above, the SODA SSH spectrum is redder than observed (left), and much mesoscale variability is simply absent in the SODA solution. Comparison of the observed and modeled tidal solution is more favorable (right). The alignment of the internal tide beam with the ground track is better, with the PEZ-HAT (solid) and observed (dashed) SSH spectra nearly overlapping over a wide range of scales. The variance levels of the mode- 1 and mode- 2 wavenumber bands are elevated and comparable in the model and observations.
The incoherent $M_{2}$ variance reaches a maximum of about one-third of the level of the coherent variance at the mode- 1 wavenumber.

Figure 7 compares observed and modeled SSH along the ground track passing through Kauai Channel, the site of several studies (Carter and Gregg 2006; Nash et al. 2006; Klymak et al. 2006; Zilberman et al. 2011). In contrast to the FFS track, the incoherent tidal variance is only about $5 \%$ of the coherent variance in the mode- 1 wave band. Spectral amplitudes associated with the internal tide agree within a factor of 2 .

\section{b. Sea surface height variability}

The surface expression of the internal tide is the result of interference from wave sources at multiple sites. Variability of the internal tide across the ensemble can be understood by considering a simple model for the wave field, which represents the surface expression of mode 1 as the sum of contributions from localized sources (Rainville et al. 2010),

$$
\eta(\mathbf{x})=A_{j}\left(\frac{\bar{r}_{j}}{r_{j}}\right)^{1 / 2} \exp \left[i\left(k_{j} r_{j}+\phi_{j}\right)\right],
$$

where $r_{j}=\left|\mathbf{x}-\mathbf{x}_{j}\right|$ is the distance from the source point $\mathbf{x}_{j}, A_{j}$ is the real-valued amplitude at distance $\bar{r}_{j}$ from the 

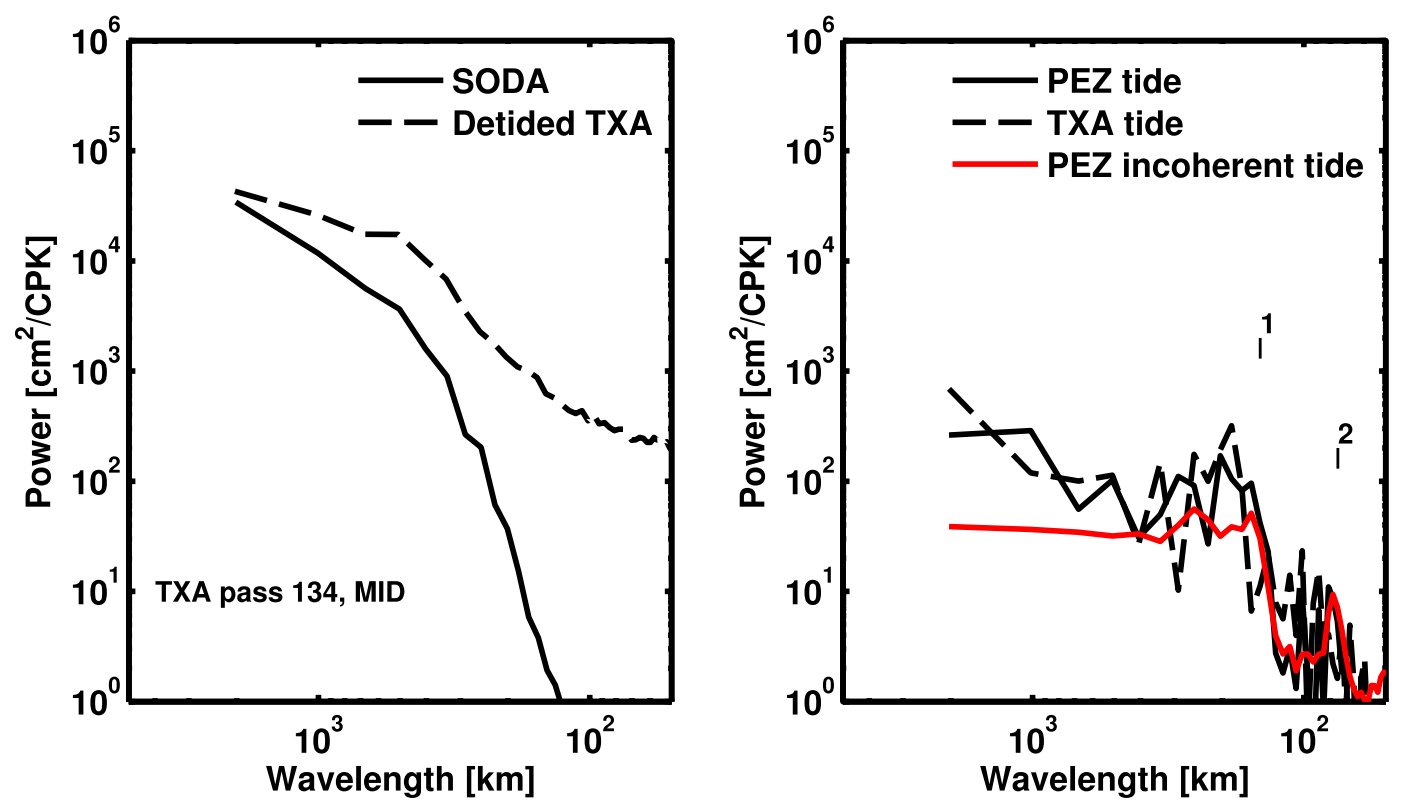

FIG. 5. Along-track wavenumber power spectrum for orbit pass 134, MID. (left) Wavenumber power spectrum for the SODA steric height relative to $2000 \mathrm{~m}$ (solid) and detided TXA observations (dash). (right) Spectrum for ensemble average of modeled (PEZ-HAT, solid) and TXA (dash) $M_{2}$ tide. Red line shows the mean power spectrum of the modeled incoherent tide, defined as the difference between the ensemble mean spectrum and the spectrum of the ensemble mean tidal SSH. Vertical line segments labeled 1 and 2 indicate the nominal wavelengths of the mode- 1 and mode-2 internal waves, respectively.

source, $\phi_{j}$ is the source phase, $k_{j}$ is the scalar wavenumber, and a sum over the repeated index $j=1, \ldots, J$ is assumed. The primary limitation of this model is the restriction to constant $k_{j}$ wavenumbers, but the along-path refraction will be considered below. Consider separately the impact of variations in source strength $A_{j}$, source location via $\bar{r}_{j}$, source phase $\phi_{j}$, and wavenumber $k_{j}$. The impact on $\eta$ can be evaluated by taking partial derivatives
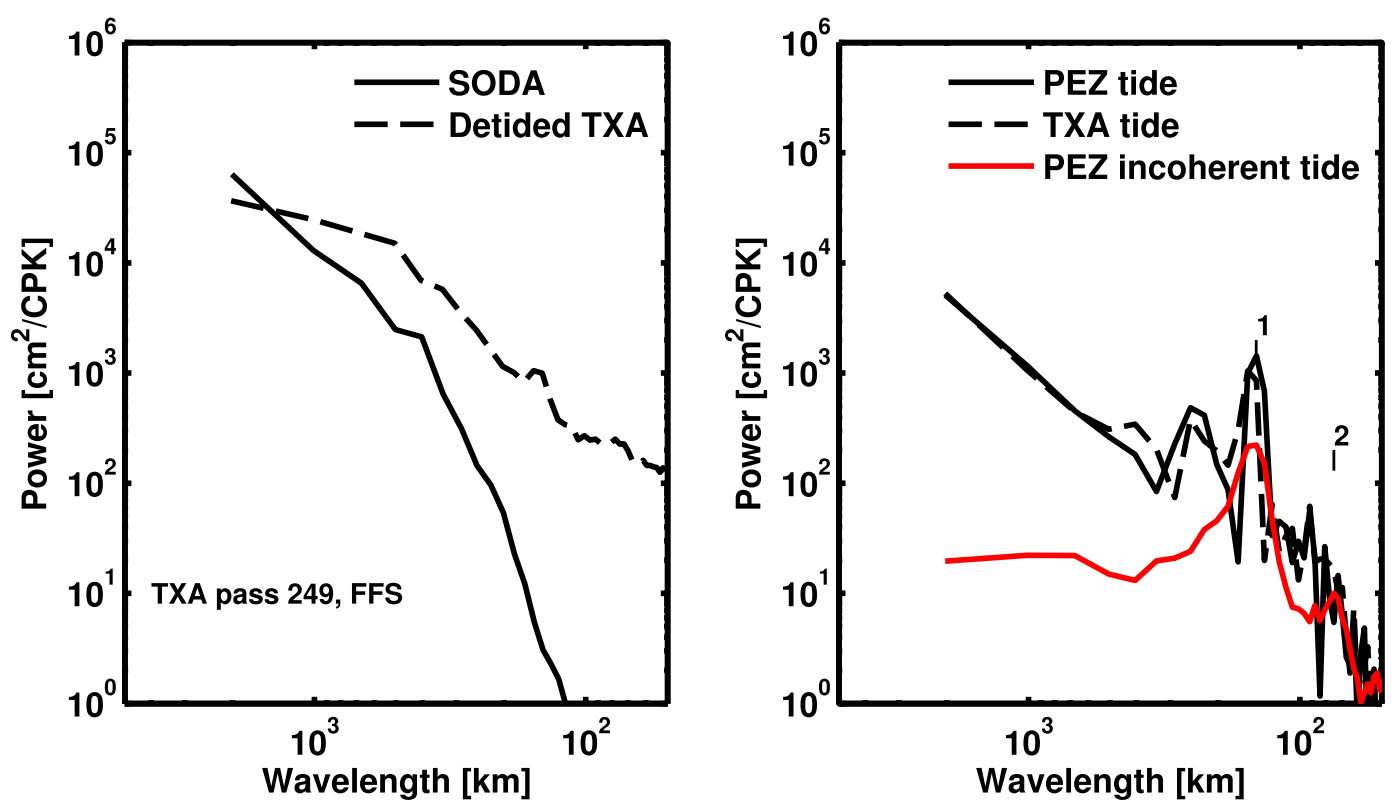

FIG. 6. As in Fig. 5, but for along-track wavenumber power spectrum for orbit pass 249, FFS. 

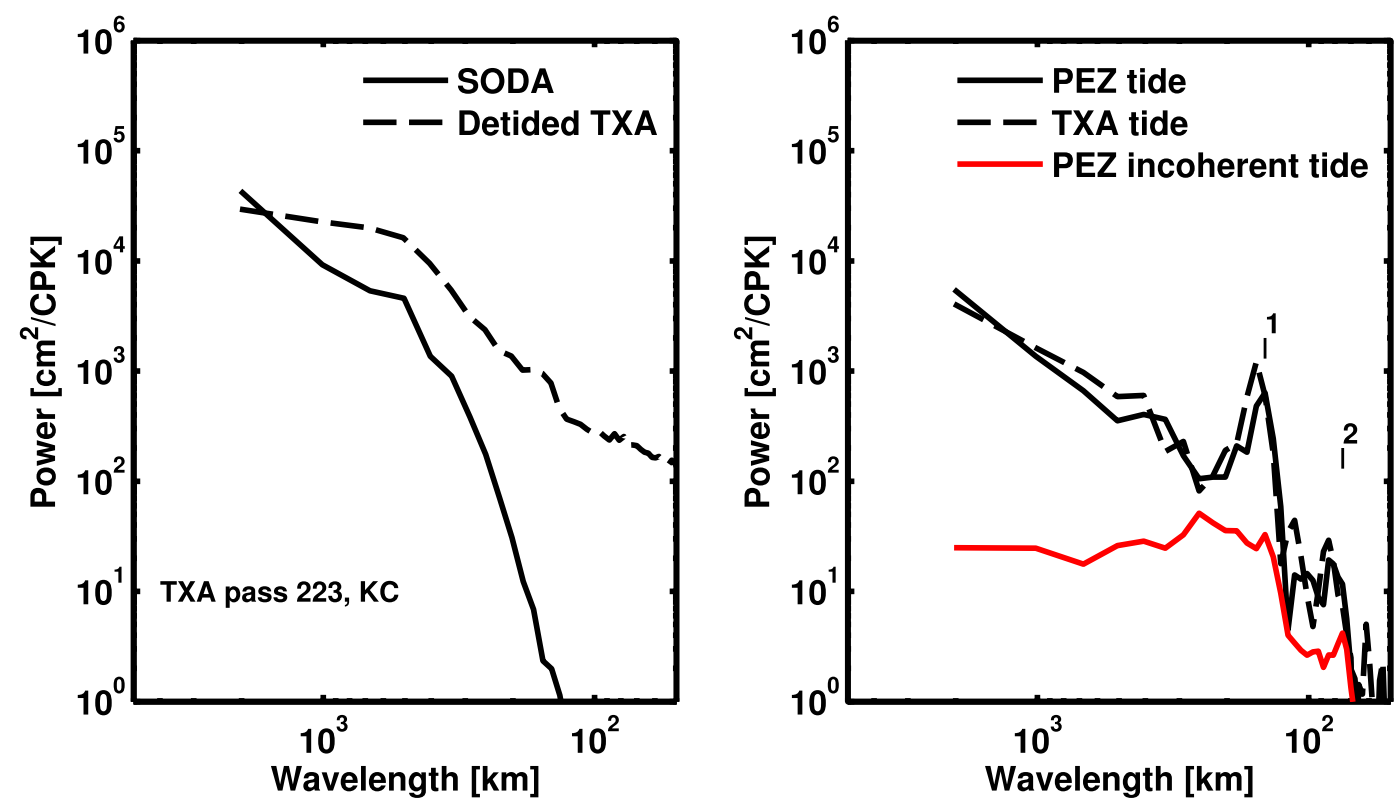

FIG. 7. As in Fig. 5, but for along-track wavenumber power spectrum for orbit pass 223, KC.

$$
\begin{aligned}
& \frac{\partial \eta}{\partial A_{j}}=\left(\frac{\bar{r}_{j}}{r_{j}}\right)^{1 / 2} \exp \left[i\left(k_{j} r_{j}+\phi_{j}\right)\right], \\
& \frac{\partial \eta}{\partial \bar{r}_{j}}=\frac{A_{j}}{2 \bar{r}_{j}}\left(\frac{\bar{r}_{j}}{r_{j}}\right)^{1 / 2} \exp \left[i\left(k_{j} r_{j}+\phi_{j}\right)\right], \\
& \frac{\partial \eta}{\partial \phi_{j}}=i A_{j}\left(\frac{\bar{r}_{j}}{r_{j}}\right)^{1 / 2} \exp \left[i\left(k_{j} r_{j}+\phi_{j}\right)\right], \quad \text { and } \\
& \frac{\partial \eta}{\partial k_{j}}=i r_{j} A_{j}\left(\frac{\bar{r}_{j}}{r_{j}}\right)^{1 / 2} \exp \left[i\left(k_{j} r_{j}+\phi_{j}\right)\right] .
\end{aligned}
$$

These expressions show that the effects of variation in $A_{j}, \bar{r}_{j}$, and $\phi_{j}$ decay with increasing distance from the source, following the $r_{j}^{-1 / 2}$ decay of the component wave field. Only changes in $k_{j}$ are capable of producing variations in $\eta$ that grow (like $r_{j}^{1 / 2}$ ) with increasing distance from the sources. Furthermore, the interference of waves from multiple sources will lead to spatial modulations in the $\eta$ variability, even for spatially constant wavenumbers.

The idealized model of Rainville et al. (2010) also included a restriction on propagation direction. In principle, changes to the azimuthal distribution of radiation from the localized sources could create $\eta$ variability that increases with distance from the sources, but it is not at all clear that the simple model is adequate to describe the azimuthal distribution of the internal waves. The azimuthal distribution of wave energy is related to the diffraction around obstacles and the geometry of the source. The internal wave generation process is most efficient where the topographic slope is equal to the slope of the wave characteristics, approximately 0.05 . This slope is so large that reasonable changes in stratification are unlikely to cause large lateral changes in generation sites which would affect the azimuthal spreading. For example, a $10 \%$ change in buoyancy frequency due to time-variable stratification would move a 700-m-deep generation site less than $2 \mathrm{~km}$. Because the dynamics of baroclinic generation can involve nonlocal contributions to the bottom pressure and mode coupling, generation site displacement could be larger than that estimated here, but the displacements are likely to be a small fraction of the mode- 1 wavelength.

Figure 8 shows the standard deviation of tidal amplitude computed from the 9-year ensemble of tidal solutions. The spatial distribution of tidal variability is consistent with a dominance of propagation path effects (variable $k_{j}$ ) over generation site effects (variable $A_{j}, \phi_{j}$, or $r_{j}$ ); variability grows with increasing distance from the ridge, rather than decaying or following the same pattern as the mean internal tide amplitude. Smallerscale spatial modulations are present because of the multipath effects caused by independent modulations of different wavenumber components.

To assess the relative contributions of processes leading to time-variable refraction, consider the phase speed of internal waves propagating through a nonuniform background; the dispersion relation is derived in the appendix. Let $c_{0}^{2}=g D_{1}$ be the squared phase speed of 


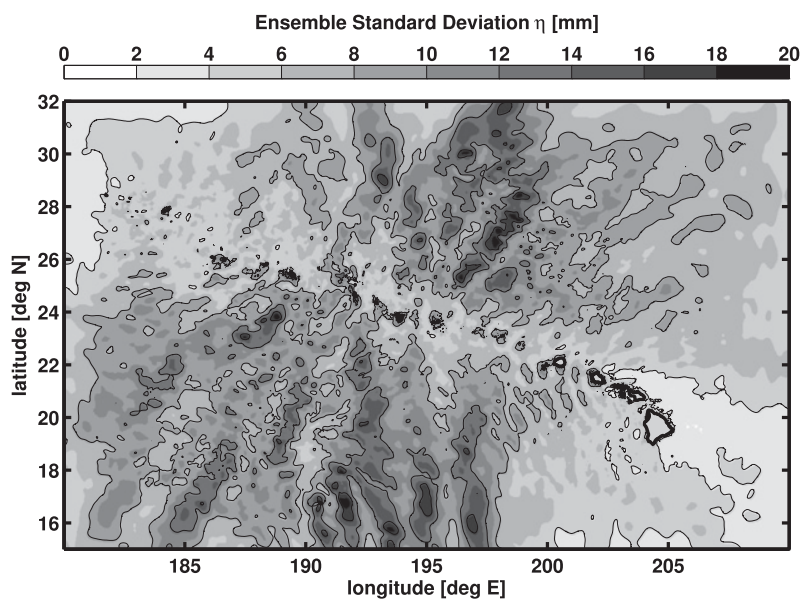

FIG. 8. The ensemble std dev of the $M_{2}$ harmonic constant.

nonrotating shallow water waves propagating in water of depth $D_{1}$ corresponding to the mode- 1 reduced depth (i.e., $c_{0}^{-2}$ is the separation constant in the mode- 1 eigenvalue problem). Relative perturbations of the phase speed $\delta c_{p} / c_{p}$ may be expressed as a sum of perturbations to $c_{0}$, Froude number $\mathrm{Fr}=|\overline{\mathbf{u}}| / c_{p}$, and relative vorticity expressed as $\operatorname{Ro}=\bar{\zeta} / f$,

$$
\frac{\delta c_{p}}{c_{p}} \approx \frac{1}{2} \frac{\delta c_{0}^{2}}{c_{0}^{2}}+\mathrm{Fr}+\frac{1}{2} \frac{f^{2}}{\omega^{2}} \mathrm{Ro},
$$

where the three terms on the right-hand side correspond to the following processes: 1) refraction due to background stratification, 2) Doppler shifting (advection by the subtidal current), and 3) refraction due to background relative vorticity (Kunze 1985). Exchanges of energy between the mean flow and waves can also occur, but these exchanges are too small to be distinguished from numerical truncation error in the present case.

Contributions to the root-mean-square phase speed perturbations $\delta c_{p} / c_{p}$ are illustrated in Fig. 9. The largest contributions are due to changes in stratification (Fig. 9a), the zonal average of which increases from south to north and approaches $5 \%$ in magnitude. The effect of variation in Froude number is somewhat smaller (Fig. 9b), and it increases to the south inversely with $f$, which controls the geostrophic current speed. Reduction in Fr near the island of Hawaii is likely a spurious artifact of the coarse resolution of SODA compared to scales of oceanic variability. The larger values of the refraction and Froude number terms over shallow topography are due to the reduction of $c_{0}$ in shallow water. The effect of relative vorticity is a factor of 10 smaller than the other terms (Fig. 9c). For geostrophic background flow these three terms are not independent, but are highly scale dependent. Scale analysis suggests that the effect of stratification will be dominant at scales larger than the Rossby radius $L_{R}=$ $c_{0} / f$; the Doppler effect will dominate at intermediate scales between $L_{R}$ and $L=c_{0} / \omega$; and relative vorticity will be the dominant effect at smaller scales.

Figure 10 illustrates some statistical characteristics of the phase speed variability. To distinguish periodic (seasonal) variability from eddies, $c_{p}$ has been decomposed into a monthly average plus an anomaly. Figure 10a shows the latitudinal dependence of the mean phase speed (heavy line) and its monthly means (thin lines). The seasonal cycle accounts for approximately $10 \%$ of the variance in $c_{p}$ (Fig. 10b). Seasonal variations are correlated over essentially the entire domain (not shown). The spatial correlation of $c_{p}$ (Fig. 10c) is dominated by the anomaly component, which has a correlation scale of about $300 \mathrm{~km}$ (full width at half maximum).

Further insight can be gained by projection of the velocity and pressure fields onto locally defined vertical modes (Griffiths and Grimshaw 2007). Based on the small size of the Rossby number, the nonlinear terms in the momentum equation are neglected; and the smallness of the Froude number compared to $\delta c_{0} / c_{0}$ suggests that convection by the subtidal flow may be neglected at leading order (Fig. 9). Neglect of these and the dissipative terms leaves a linear system that incorporates the effects of refraction by the background flow and topographic scattering. The linear Boussinesq shallow water equations take the form

$$
\begin{aligned}
& -i \omega \hat{\mathbf{u}}^{(n)}+f \mathbf{k} \times \hat{\mathbf{u}}^{(n)}+g \nabla \hat{p}^{(n)} \\
& \quad=-g D_{n} \sum_{m} I_{n m} \hat{p}^{(m)} \nabla H+\cdots \quad \text { and } \\
& -i \omega \hat{p}^{(n)}+D_{n} \boldsymbol{\nabla} \cdot \hat{\mathbf{u}}^{(n)} \\
& \quad=-D_{n} \sum_{m} I_{n m} \hat{\mathbf{u}}^{(m)} \cdot \nabla H+\cdots,
\end{aligned}
$$

where modal coupling coefficients $I_{n m}$ are equal to $I_{n m}=D_{n} /\left(D_{n}-D_{m}\right)\left[\phi^{(m)} \phi^{(n)}\right]_{z=-H}$ for $n \neq m$ and $I_{n m}=\left[\phi^{(m)} \phi^{(n)}\right]_{z=-H} / 2$ for $n=m$, and dots denote omitted terms arising from nonlinearity, dissipation, and the astronomical tide-generating force. The horizontal velocity vector $\mathbf{u}=(u, v)$ and the pressure $p$ have been expanded in terms of vertical modes

$$
\{u, v, p\}=\sum_{n}\left\{\hat{u}^{(n)}, \hat{v}^{(n)}, \hat{p}^{(n)}\right\} \phi^{(n)}(z ; x, y),
$$

where curly brackets enclose the set of three functions, and the modal amplitudes $\left\{\hat{u}^{(n)}, \hat{\boldsymbol{v}}^{(n)}, \hat{p}^{(n)}\right\}$ are a function only of the horizontal coordinates $(x, y)$. The vertical mode functions $\phi^{(n)}$ are an explicit function of the vertical coordinate $z$, but they depend implicitly on $(x, y)$ 

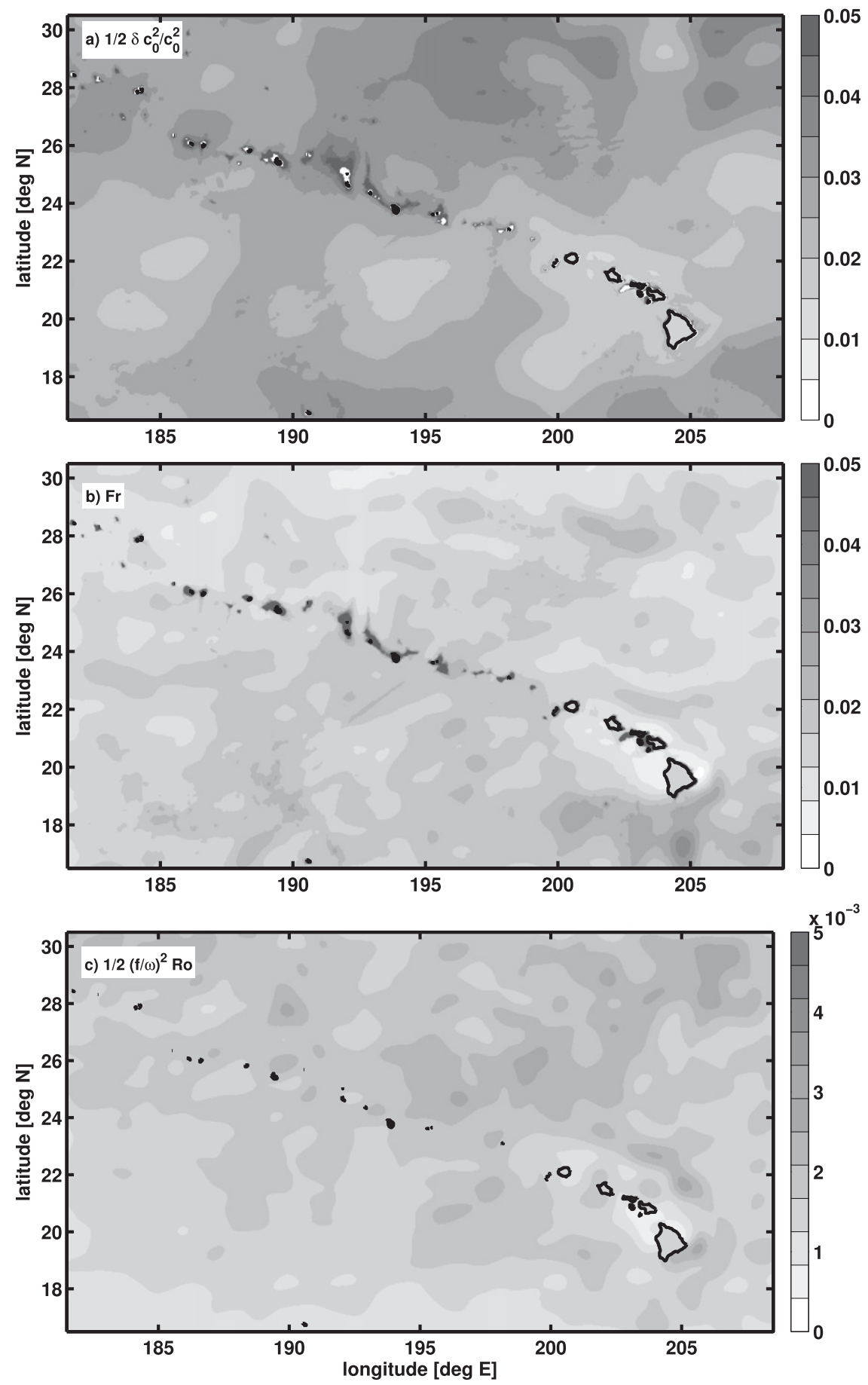

FIG. 9. Normalized contributions to mode-1 time-variable refraction. (a) Std dev of mode-1 eigenvalue $c_{0}^{2}=g D$ is a direct effect of time-variable stratification. (b) Std dev (averaged over the top $1000 \mathrm{~m}$ ) of $\mathrm{Fr}=|\overline{\mathbf{u}}| c_{p}$ is the effect of time-variable Doppler shift. (c) Root-mean square of the scaled Ro $=\bar{\zeta} / f$ is the effect of relative vorticity (note different scale compared to upper panels). 

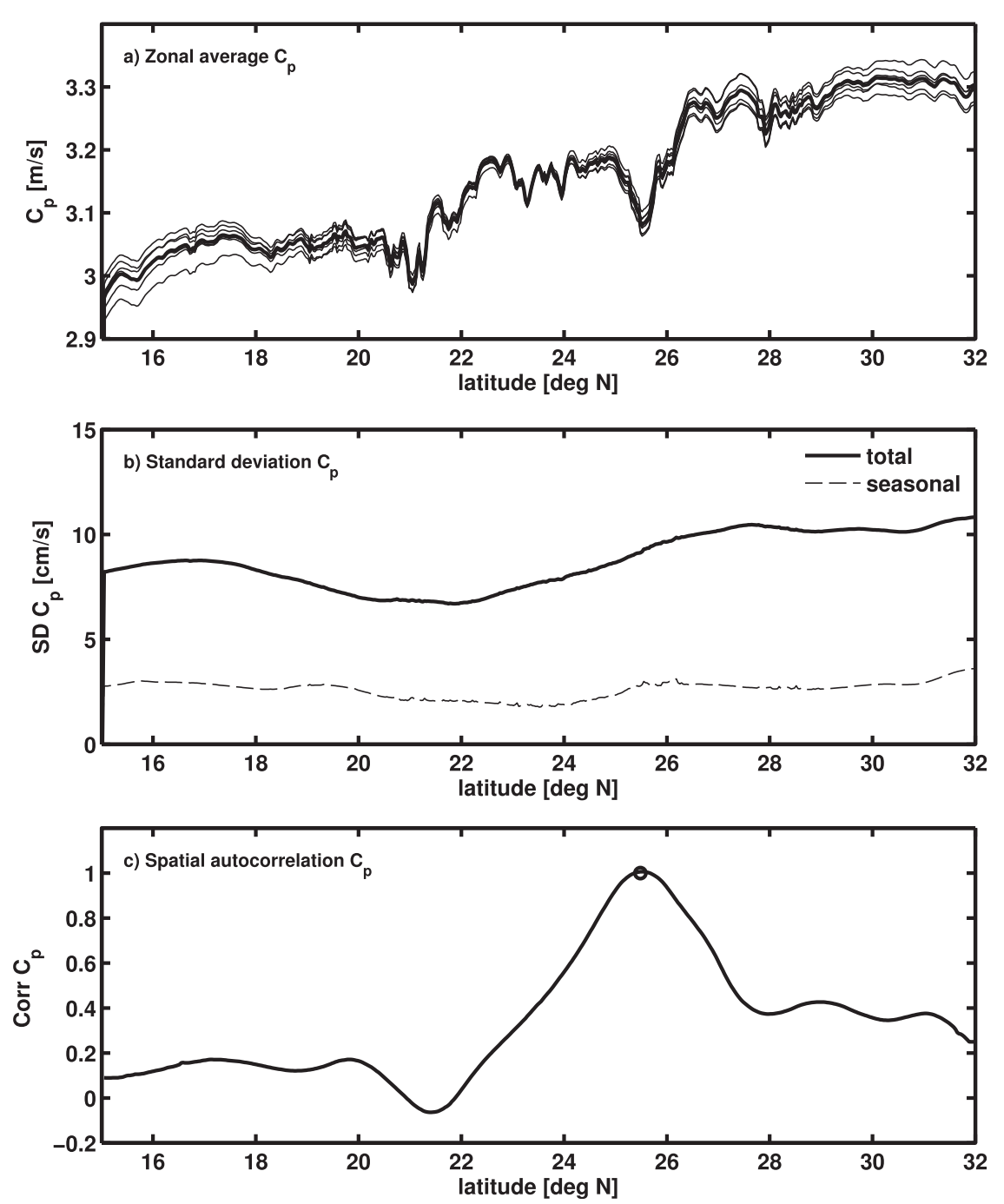

FIG. 10. Statistics of mode-1 phase speed. (a) Zonal average of mean mode- 1 phase speed (thick line) and average within specific months (thin lines). (b) Std dev of phase speed (solid) and the seasonally variable part (dash), as defined by the monthly averages. (c) A zonal slice of the spatial autocorrelation function of the mode- 1 phase speed. Correlation is with respect to the MP1 site $\left(25.5^{\circ} \mathrm{N}, 194.9^{\circ} \mathrm{E}\right)$.

through their dependence on water depth and the background buoyancy profile $N^{2}(x, y, z)=-g \bar{\rho}_{z} / \rho_{0}-\left(g / c_{s}\right)^{2}$, where $c_{s}$ is the sound speed. The mode functions $\phi^{(m)}=\rho_{0} g D_{m} \zeta_{z}^{(m)}$ are obtained via solutions of the eigenvalue problem (Hendershott 1981)

$$
\zeta_{z z}^{(m)}+\frac{N^{2}}{g D_{m}} \zeta^{(m)}=0,
$$

for $z \in(0,-H)$, with boundary conditions $\zeta^{(m)}(z)=0$ at $z=-H$, and $\zeta^{(m)}-D_{m} \zeta_{z}^{(m)}=0$ at $z=0$. The equivalent depths $D_{m}$ are computed directly from the eigenvalues of this system. The notation for the modal coupling terms in Eqs. (7) and (8), $\left[\phi^{(m)} \phi^{(n)}\right]_{z=-H}$, denotes the product of vertical modes evaluated at the bottom $z=-H$. Because the pressure variable has not been eliminated, Eqs. (7) and (8) appear simpler than those in Griffiths and Grimshaw (2007); although, they are derived following the same methodology.

Equations (7) and (8) are written in a form that exposes their similarity to the shallow water equations for a homogenous layer of fluid. The system thus supports a spectrum of Poincaré- and Kelvin-like waves modified by both refraction (spatially variable $g D_{n}$ ) and mode coupling. Sea surface height $\eta$ is related to the pressure at the surface, $p(z=0)=\rho_{0} g \eta$, which permits the sea surface height to be decomposed into a sum of modal contributions, $\eta=\sum_{m} \eta^{(m)}$, where $\eta^{(m)}=\hat{p}^{(m)} /\left(\rho_{0} g\right)\left[\phi^{(m)}\right]_{z=0}$. 


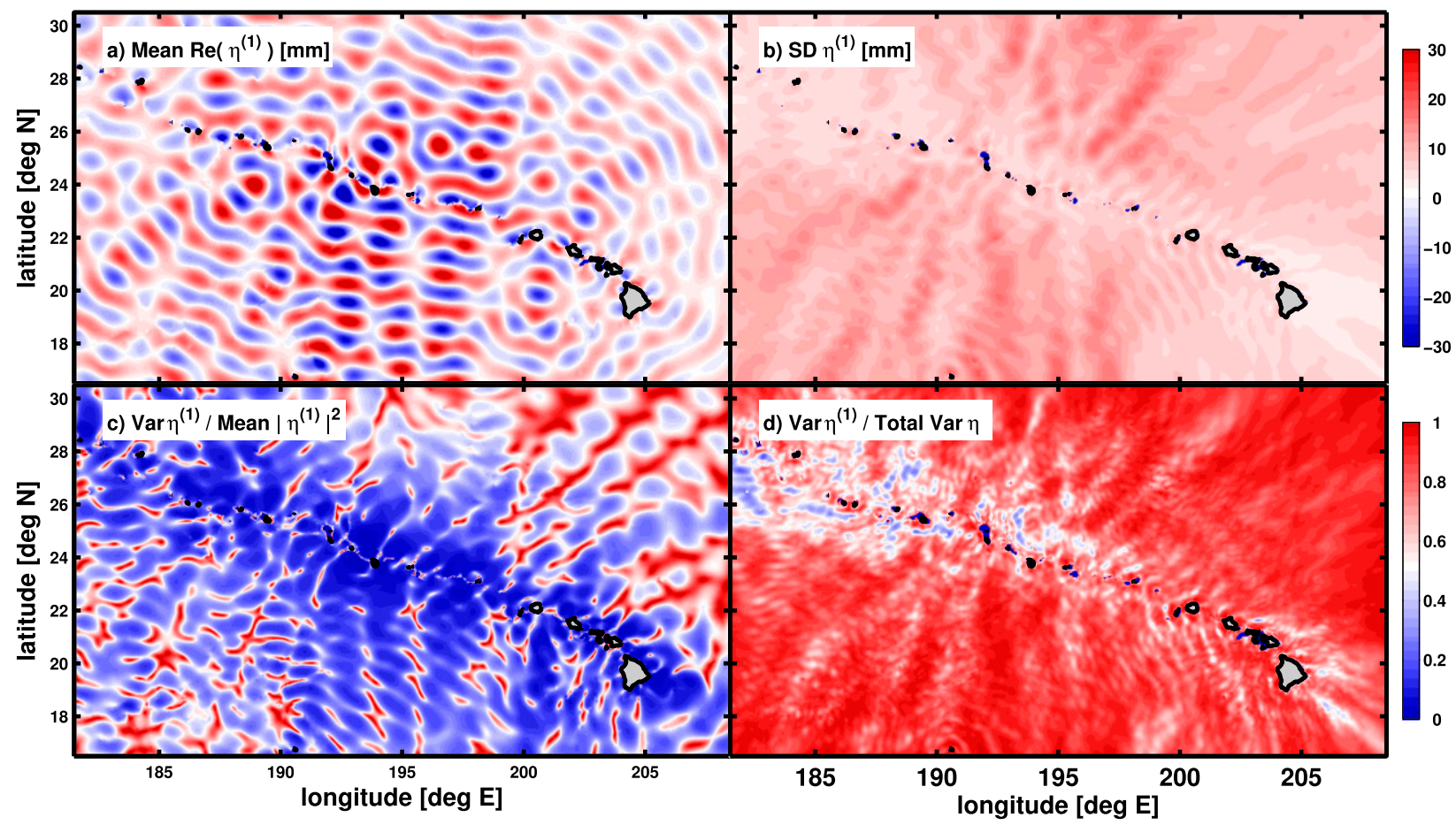

FIG. 11. Mode-1 ensemble mean and variability. (a) The real part (in phase) of the mode-1 ensemble mean sea surface expression has peak amplitudes from 3 to $4 \mathrm{~cm}$. (b) The std dev of mode- 1 ensemble attains a max value of nearly $2 \mathrm{~cm}$ at distance from the ridge. (c) The incoherent fraction of the mean-squared mode-1 signal is spatially variable, consistent with the along-track analysis in Figs. 5-7. (d) Mode1 variability is the largest part of total tidal variability (all modes) over most of the domain. Higher than mode- 1 variability is significant closer to the ridge, particularly to the northeast and in between mode-1 "beams."

Thus, one can interpret the variability of $\eta$ in terms of the surface expression of the pressure modes. Equation (8) shows that variability in $\hat{p}^{(m)}$ is the combined result of local changes in stratification, which determines $D_{n}$ and $\left[\phi^{(m)} \phi^{(n)}\right]_{z=-H}$, and nonlocal changes in generation and propagation, which determine $\hat{\mathbf{u}}^{(n)}$. Using the WentzelKramers-Brillouin (WKB) approximation to estimate the solution of Eq. (10), one finds that the equivalent depth $D_{n}$ depends only on the vertical integral of the buoyancy frequency

$$
D_{n} \approx\left[\int_{-H}^{0} N(z) d z\right]^{2} /\left(g n^{2} \pi^{2}\right)
$$

The mode functions at the ocean surface and bottom $\left[\phi^{(m)}\right]_{z=0,-H}$, are proportional to $\left(N D_{n}\right)^{1 / 2}$ evaluated at the same depths. Changes in the buoyancy frequency $N^{1 / 2}$ at the ocean bottom, $z=-H$, are very small; hence, time variability in $\left[\phi^{(m)} \phi^{(n)}\right]_{z=-H}$ is due to variability in $D_{n}$, and the fractional variation is the same as displayed in Fig. 9a.

Figure 11 displays the internal mode- 1 surface elevation field $\eta^{(1)}$ along with several metrics of its variability. The real part (in-phase component) of the ensemblemean $\eta^{(1)}$ (Fig. 11a) illustrates the spatially variable wave field that results from interference of multiple sources along the ridge. The standard deviation of $\eta^{(1)}$ (shown in Fig. 11b with the same color scale as in Fig. 11a) mimics most aspects of Fig. 8 and shows the increase of variability with distance from the ridge modulated by smaller-scale spatial fluctuations that result from interference. In particular, note that the bottom topography field $(|\nabla H|)$ is not evident in the field of $\eta^{(1)}$ variability, as would be the case if variability in the modal coupling terms were significant. Figure 11c illustrates the variance of the mode- 1 height as a fraction of the mean, which would be associated with the incoherent tide. Close to the ridge the variance is a small fraction of the mean-square modal amplitude, except near nodal lines in the $\eta^{(1)}$ field. Because mode 1 has the largest sea surface expression of the internal modes, its variability is responsible for the majority of the tidal variability, due to all modes (Fig. 11d). The degree to which detection of the nonstationarity mode- 1 internal tide depends on the location and orientation of the measurement array is apparent from Fig. 11b, which shows more than a factor of 2 differences in the standard deviation depending on location. The spatial variability in the coefficient of variation (Fig. 11c) is even more extreme because of the large relative values in the nodal areas between beams where the mean amplitude is small. 


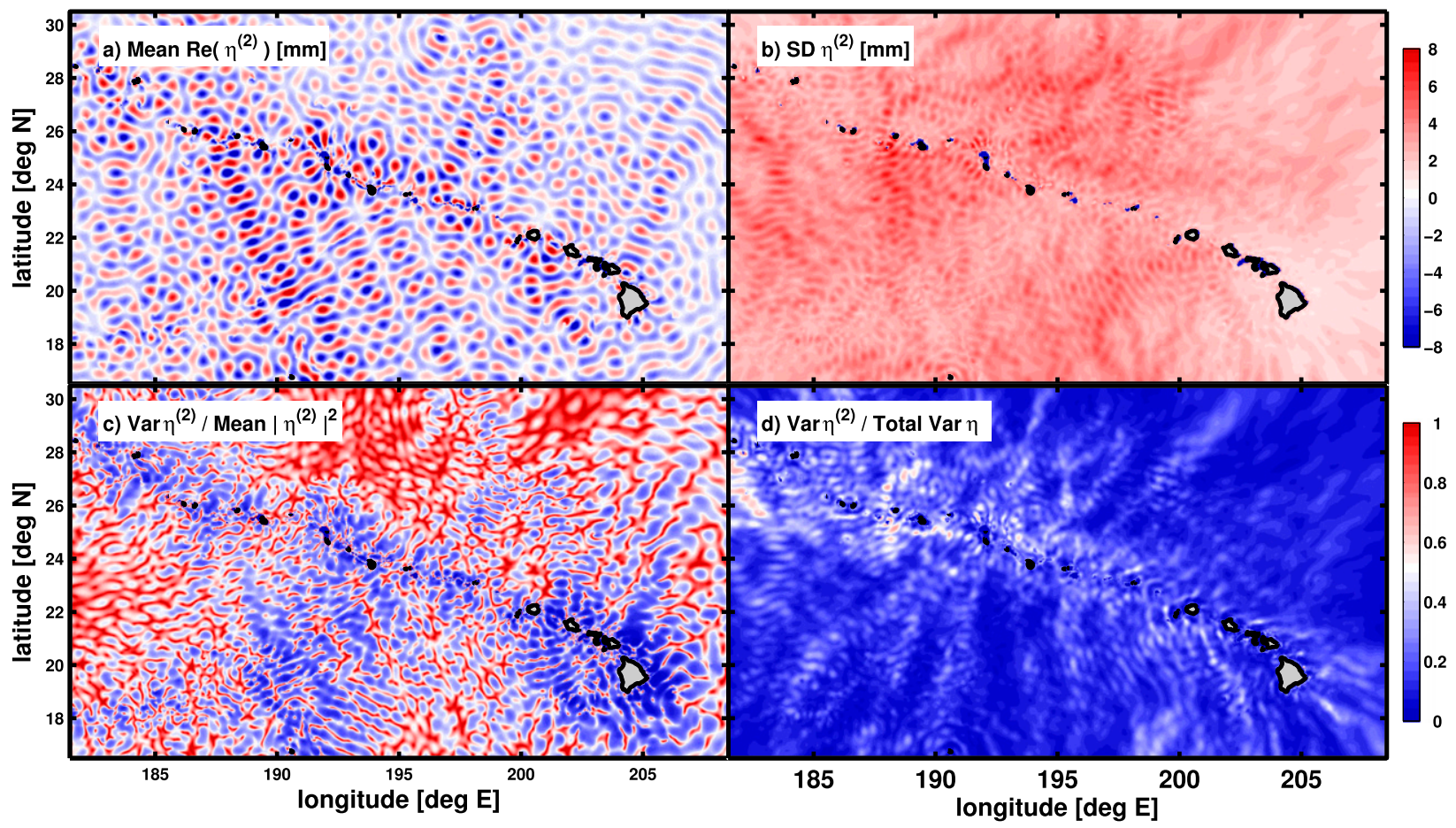

FIG. 12. Mode-2 ensemble mean and variability. See Fig. 11 caption for description. Note different color scale compared to Fig. 11a,b.

Figure 12 displays the same set of metrics for the second internal mode. Note that the phase speed of mode 2 is approximately on-half of that of mode 1 , thus the effect of Froude number on mode 2 is twice as large as shown in Fig. 9b. The mode- 2 signal is more spatially complex than mode 1 because of its shorter wavelength, although the amplitude of the signal is only about onequarter of that of mode 1 . The greatest mode- 2 variability is associated with the generation sites at the Line Islands. Interpretation of the fractional variability in Fig. $12 \mathrm{c}$ is complicated by small scale of the mode- 2 waves; however, the fractional variability of mode 2 is higher than mode 1 , as would be expected.

The above observations suggest that the primary effect of the subtidal fields is to modify the propagation of the internal tide, rather than its generation. Is it possible to reconcile the quantitative measure of tidal variability $(25 \%-50 \%$ coefficient of variation at 2 wavelengths from the ridge; cf. Fig. 8 or 11b) with phase speed variability ( $5 \%$ coefficient of variation; cf. Fig. 9)? Consider the phase of an individual wave component which has traveled distance $r$ along a ray path. The phase is given by $\phi(r)=\phi_{0}+\int_{0}^{r} k\left(r^{\prime}\right) d r^{\prime}$, where $\phi_{0}$ is the phase at $r=0$, and $k(r)$ is wavenumber. A straightforward calculation finds the phase variance is given by

$$
[\delta \phi(r)]^{2}=\bar{k}^{2}\left(\frac{\delta c_{p}}{\bar{c}_{p}}\right)^{2} \int_{0}^{r} \int_{0}^{r} R\left(r^{\prime}-s^{\prime}\right) d r^{\prime} d s^{\prime},
$$

where $\delta \phi$ denotes the standard deviation of $\phi$, and $R(s)$ is the spatial autocorrelation function of $c_{p}$, which is assumed to be homogeneous along the ray path. Using Fig. 10 as a guide, one can approximate $R$ with a bellshaped kernel, $R(s)=\exp (-s / L)^{2}$, with $L=180 \mathrm{~km}$ (corresponding to the $300-\mathrm{km}$ full width at half maximum previously mentioned), and estimate the phase variance as

$$
[\delta \phi(r)]^{2} \approx \sqrt{\pi} L r \bar{k}^{2}\left(\frac{\delta c_{p}}{\bar{c}_{p}}\right)^{2} .
$$

Taking $\delta c_{p} / \bar{c}_{p}=0.05$ and $\bar{k}=2 \pi / 180 \mathrm{~km}$, one obtains $[\delta \phi(r)]^{2} \approx 0.03 r / L$. For Gaussian phase fluctuations, the expected value of $\exp (i \phi)$ is $\exp \left\{-[\delta \phi(r)]^{2}\right\}$ (Middleton 1960). The coefficient of variation of $\eta$ due to phase variability is thus $\left(1-\exp \left\{-[\delta \phi(r)]^{2}\right\}\right)^{1 / 2} \approx \delta \phi(r)$. Inserting $r / L=2$, one finds the incoherent amplitude to be $24 \%$ of the mean amplitude after the mode- 1 tide has traversed two wavelengths. More precise estimates are difficult because of multipath effects and inhomogeneity, but we conclude that the level of tidal variability is consistent with the $5 \%$ phase speed variability in SODA.

\section{c. Internal tide energetics}

In the model it is possible to unambiguously diagnose the energetics of the internal tide, a task that is difficult to do with observations because the baroclinic and 


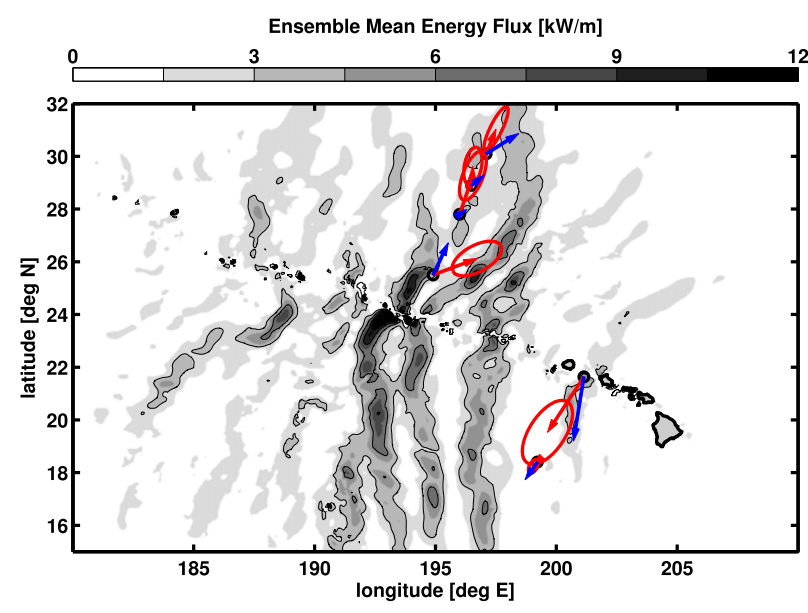

FIG. 13. The amplitude of the ensemble-mean internal wave energy flux. Arrows indicate observed (black) and modeled (red) energy flux vectors at sites noted in Fig. 1. Ellipses (red) are aligned with the principle axes of variability of the energy flux in the ensemble, with size indicating 95 percentile limits assuming normality. See Table 1 for a quantitative comparison between observed and modeled fluxes.

barotropic components of the pressure cannot usually be unambiguously separated, particularly over regions of sloping topography where internal tide generation occurs. Define the vector internal wave energy flux

$$
\mathbf{F}=\int_{-H}^{0} \mathbf{u}^{\prime} p^{\prime} d z
$$

where $H$ is the water depth, $\mathbf{u}^{\prime}$ is the baroclinic velocity, and $p^{\prime}$ is baroclinic pressure, the latter two quantities being defined as perturbations from vertical averages. Alternative definitions of the energy flux are possible (Kelly et al. 2010), but these are not used here to maintain compatibility with the definitions of the energy flux computed from the observed data. In any event, removal of the pressure perturbation associated with the surface tidal heaving, as recommended by Kelly et al. (2010), leads to insignificant corrections at the deep sites considered.

Figure 13 shows the ensemble average $|\mathbf{F}|$ over the model domain. One sees the pattern of internal wave "beams" emanating from generation sites, FFS being the largest of these with a maximum energy flux of about $20 \mathrm{~kW} \mathrm{~m}^{-1}$ to the south. Selected vectors are shown for the model (red) and observations (blue) at sites indicated in Fig. 1. Red ellipses are aligned with the principle axes of variability of the energy flux, scaled by 1.96 to represent the 95 percentile limits of variation, assuming normality.

Figure 14 shows the PEZ-HAT energy flux vectors at the FLIP and MP1 sites for each member of the ensemble (gray arrows), as well as the ensemble mean (black arrow), and the ellipse of variation (dot-dash line). The observed flux at FLIP is slightly larger than the largest flux obtained in the ensemble, aligned in nearly the same direction (cf. Fig. 13 and Table 1). The observed flux at the MP1 site lies within the range of amplitudes obtained in the ensemble, but it is oriented about $25^{\circ}$ counterclockwise from the outer limit of the variability ellipse. Table 1 summarizes the observed and computed energy fluxes at all five sites. The observed and modeled fluxes are quite different, but considering the spatial variability (e.g., Fig. 13) and temporal variability (e.g., Fig. 14) of the flux vectors, the disagreement with observations is unsurprising. The flux at MP1-MP4 sites is also influenced by waves propagating southward from the Aleutians (Zhao et al. 2010), which are not included in the model.
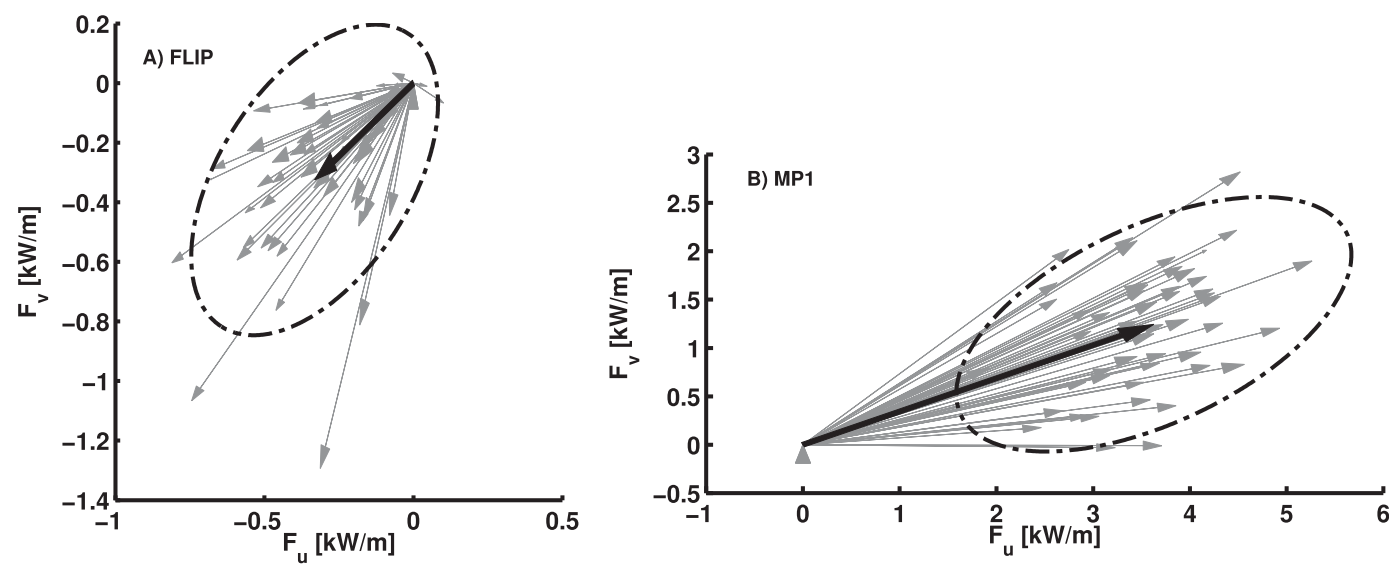

FIG. 14. The ensemble mean (black) and each realization (gray) of the $M_{2}$ internal wave energy flux at (a) FLIP and (b) MP1 sites. Dot-dash lines indicate the $95 \%$ variability ellipse. 
TABLE 1. Internal wave energy flux.

\begin{tabular}{|c|c|c|c|c|c|}
\hline \multirow[b]{2}{*}{ Site } & \multirow[b]{2}{*}{ Location } & \multicolumn{2}{|r|}{ Observation } & \multicolumn{2}{|r|}{ Model } \\
\hline & & $\mathrm{kW} \mathrm{m}^{-1}$ & $\begin{array}{c}\text { Direction, counterclockwise } \\
\text { from east }\end{array}$ & $\mathrm{kW} \mathrm{m}^{-1}$ & $\begin{array}{c}\text { Direction, counterclockwise } \\
\text { from east }\end{array}$ \\
\hline $\mathrm{C} 2^{\mathrm{a}}$ & $21.6^{\circ} \mathrm{N}, 201.1^{\circ} \mathrm{E}$ & 4.96 & $260.0^{\circ}$ & 5.19 & $234.5^{\circ}$ \\
\hline FLIP $^{\mathrm{b}}$ & $18.4^{\circ} \mathrm{N}, 199.2^{\circ} \mathrm{E}$ & 1.70 & $232.8^{\circ}$ & 0.47 & $223.7^{\circ}$ \\
\hline $\mathrm{MP}^{\mathrm{c}}$ & $25.5^{\circ} \mathrm{N}, 194.9^{\circ} \mathrm{E}$ & 2.76 & $62.5^{\circ}$ & 3.82 & $18.9^{\circ}$ \\
\hline$M P 2^{c}$ & $27.8^{\circ} \mathrm{N}, 196.0^{\circ} \mathrm{E}$ & 0.71 & $31.3^{\circ}$ & 3.12 & $70.2^{\circ}$ \\
\hline $\mathrm{MP}^{\mathrm{c}}$ & $28.9^{\circ} \mathrm{N}, 196.5^{\circ} \mathrm{E}$ & 1.34 & $36.5^{\circ}$ & 1.51 & $85.1^{\circ}$ \\
\hline $\mathrm{MP}^{\mathrm{c}}$ & $30.1^{\circ} \mathrm{N}, 197.1^{\circ} \mathrm{E}$ & 3.10 & $29.8^{\circ}$ & 2.05 & $66.9^{\circ}$ \\
\hline
\end{tabular}

${ }^{\mathrm{a}}$ Zilberman et al. (2011).

${ }^{\mathrm{b}}$ Rainville and Pinkel (2006a).

${ }^{\mathrm{c}}$ Zhao et al. (2010).

Because the wave energy flux is a quadratic quantity, the ensemble-mean flux differs from the flux of the ensemble mean. The former quantity is the average energy flux of the coherent and incoherent internal waves, while the latter is due to the coherent waves alone. Letting $\mathbf{F}$ denote the mean flux, and $\overline{\mathbf{F}}$ denote the flux of the mean fields, the divergence of their difference, $D=\nabla(\mathbf{F}-\overline{\mathbf{F}})$ is the rate of energy conversion, or scattering, of the coherent into incoherent tide (Fig. 15). A $D>0$ occurs where the internal tide is scattered by time variations of the subtidal background field, leading to conversion rates of $152 \mathrm{~mW} \mathrm{~m}^{-2}$ at several sites. For comparison, models indicate that peak rates of barotropic-tobaroclinic conversion are in excess of $2 \mathrm{~W} \mathrm{~m}^{-2}$ at sites such as FFS and KC. Here, $D<0$ occurs at sites where energy is added to the coherent internal tide in excess of scattering to the incoherent tide; such as typically occurs at sites of barotropic-to-baroclinic generation as just

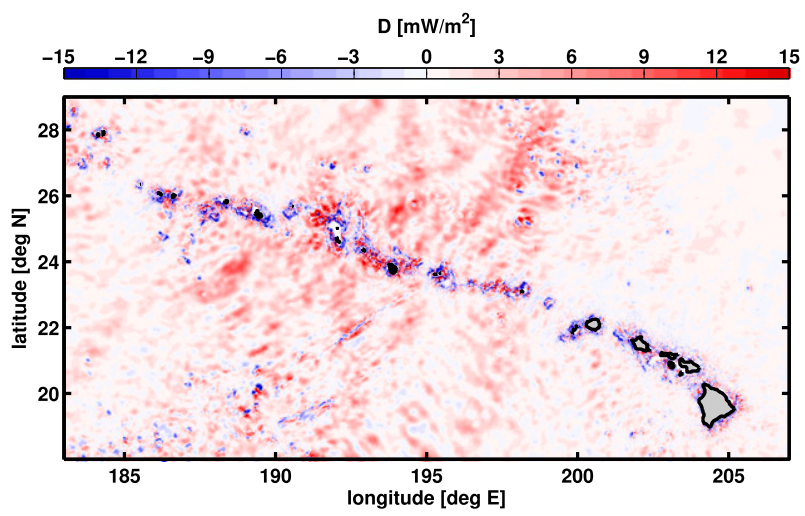

FIG. 15. Divergence of total minus coherent energy flux. Red shading $(D>0)$ shows the rate of conversion of coherent-toincoherent internal wave energy. Blue areas $(D<0)$ are sites of barotropic-to-baroclinic conversion, which predominantly pumps energy into the coherent internal wave field. Note that values of the latter exceed $2 \mathrm{~W} \mathrm{~m}^{-2}$ at tidal conversion "hot spots," such as FFS and $\mathrm{KC}$, and appear as small-scale blue/black features at the lower limit of the color scale. mentioned. It is interesting to note that the coherentto-incoherent energy transfer is spatially variable, with no simple relationship to the Froude number, Rossby number, or background buoyancy fluctuations. To the north of the ridge, incoherent scattering is associated with bottom roughness elements in the Musicians Seamount province (Rea and Naugler 1971), where higher modes are generated and de-phased by the subtidal background. French Frigate Shoals and Maru Reef, to its west, are also prominent sites of incoherent generation.

In contrast to the $\eta$ variability, which is dominated by propagation path effects, considerable variability of the energy flux divergence occurs at both generation sites and at distance from the ridge. Interestingly, the net barotropic-to-baroclinic conversion, as measured by the flux normal to the ridge at a distance of $250 \mathrm{~km}$ from the nominal ridge axis, is relatively invariant. Figure 16 illustrates the net conversion for each member of the ensemble, which has a standard deviation of $0.3 \mathrm{GW}$. In contrast, the difference between the mean total flux $(7.2 \mathrm{GW})$ and the coherent-only flux $(6.0 \mathrm{GW})$ is $1.2 \mathrm{GW}$. Thus, the variability in barotropic-to-baroclinic conversion integrated along the ridge is a small fraction of the mean scattering to the incoherent tide.

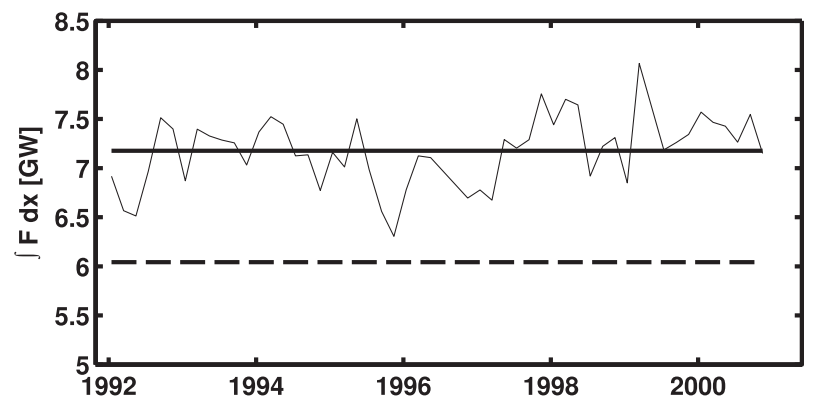

FIG. 16. Instantaneous (thin solid), ensemble-average (heavy solid), and coherent (dash) baroclinic energy flux from the ridge at a distance of $250 \mathrm{~km}$ from the nominal ridge axis. Ridge-normal flux based on the coherent tide underestimates the mean flux by about $1.2 \mathrm{GW}$, or almost $20 \%$ 
In closing, one aspect of Fig. 15 deserves a comment. Note the vastly different scales at which energy is input into the internal tide $(4 \mathrm{~km})$, the wavelength of the energy-containing baroclinic tide $(150 \mathrm{~km})$, and the inhomogeneities due to distinct generation sites and variations in the general circulation $(1000 \mathrm{~km})$. Accurately representing the generation and propagation of even the low-mode, quasi-linear, internal tide requires accurately modeling a large area at very high resolution.

\section{Discussion}

Baroclinic tides and energy fluxes are highly variable when measured at particular sites (e.g., Wunsch 1975; Zilberman et al. 2011). For example, the latter paper found changes of $20 \%$ in perturbation bottom pressure, which led to changes in excess of $40 \%$ in inferred barotropic-to-baroclinic conversion and baroclinic energy flux; values that appear much larger than those in PEZ-HAT. To compare observations with the present results, it is necessary to compare variability with the same metric. The standard deviation and coefficient of variation are emphasized here, while range of values is used in many observational studies. For sinusoidal variations, the peak-to-peak range is reduced by a factor of $(2 \sqrt{2})^{-1}$ when converting to the standard deviation. A detailed comparison at the $\mathrm{C} 2$ site (Zilberman et al. 2011) finds that the standard deviation of the baroclinic energy flux is $870 \mathrm{~W} \mathrm{~m}^{-1}$, about $190 \mathrm{~W} \mathrm{~m}^{-1}$ less than the standard deviation inferred from the peak-to-peak range at the site. Barotropic-to-baroclinic conversion has also been computed at the $\mathrm{A} 2$ site located on the steep slope $17 \mathrm{~km}$ northeast of the $\mathrm{C} 2$ site (Zilberman et al. 2011), and a standard deviation of $0.152 \mathrm{~W} \mathrm{~m}^{-2}$ is found, which is not significantly different from the observed variability. Thus, while the $\eta$ variability is generally low near the ridge, the variability of energy flux and barotropic-to-baroclinic conversion is consistent with observations, particularly given the uncertainty due to the short duration of observations.

In contrast to mooring-based observations, inferences from long-range reciprocal acoustic travel time (Dushaw et al. 1995) and along-track satellite altimetry (Dushaw et al. 2011; Ray and Zaron 2011) find that much of the baroclinic tidal variance is coherent and phase locked with its forcing. The maps presented in Figs. 8, 11, and 12 illustrate the spatial inhomogeneity of tidal variability and suggest that some caution is warranted in interpreting along-track or line-integral measurements.

Using a data-assimilative barotropic tidal model, Zaron and Egbert (2006a) concluded that energy is lost from the $M_{2}$ barotropic tide at a rate of $18-25 \mathrm{GW}$ along the Hawaiian Ridge. When extrapolated to the entire ridge, microstructure turbulence measurements (Klymak et al. 2006, 2008) and regional numerical models (Carter et al. 2008) indicate that $10 \%-20 \%$ of the energy lost from the barotropic tide is dissipated near the ridge, leaving at least $16-21 \mathrm{GW}$ to propagate away. Recent altimeter-based estimates find approximately $6.7 \mathrm{GW}$ of coherent mode- $1 M_{2}$ baroclinic energy radiated from the ridge (Zhao et al. 2011). When a plausible estimate for the higher than mode- 1 fluxes are included $(10 \%-$ 40\%; Lee et al. 2006; Rainville and Pinkel 2006a; Alford et al. 2007; Zhao et al. 2010), the $M_{2}$ energy flux is in the range of $7-9 \mathrm{GW}$. Thus, there is a difference of 7-11 GW between these two estimates. Can the incoherent baroclinic energy flux explain this difference when averaged over the ridge? Figure 16 indicates that the ensemble-average baroclinic energy flux away from the ridge is about $20 \%$ larger than the energy flux owing to the coherent part alone, where the ridge-normal flux is integrated over a 500-km-wide patch aligned with the nominal ridge axis [the same patch used by Klymak et al. (2006) and for the above-quoted flux from Zhao et al. (2010)]. The apparent energy loss due to scattering by time-variable refraction in PEZ-HAT is thus somewhat smaller than the tidally driven dissipation inferred from microstructure turbulence measurements, and it is not sufficient to explain the difference between barotropic tide energetics and the observed coherent internal tide energy flux.

From the rate of conversion of coherent-to-incoherent tidal energy in Fig. 15, one may estimate the contribution of the internal tides to mixing of the deep ocean. If it is assumed that the energy in the incoherent tide rapidly enters the broadband internal wave spectrum, the energy conversion rate can be equated with the kinetic energy dissipation rate, and the tidal contribution to the turbulent diffusivity can be inferred. The domain-average rate of coherent-to-incoherent conversion is $1.6 \mathrm{~mW} \mathrm{~m}^{-2}$ for all sites where $D>0$. Although small, this value is at least 10 times the direct dissipation rate of the baroclinic tide away from the generation sites, as computed by numerical models (Carter et al. 2008). If this energy were lost to turbulence in the upper $1000 \mathrm{~m}$ of the water column, the corresponding dissipation would be $\epsilon=2 \times 10^{-9} \mathrm{~W} \mathrm{~kg}^{-1}$, which is equivalent to a turbulent diffusivity of $K_{\rho}=$ $1.3 \times 10^{-5} \mathrm{~m}^{2} \mathrm{~s}^{-1}$, where a value of squared buoyancy frequency $N^{2}=3 \times 10^{-5} \mathrm{~s}^{-2}$ has been assumed (Osborn 1980). In some areas the conversion rate is ten times larger, indicating a local contribution to $K_{\rho}$ greater than $10^{-4} \mathrm{~m}^{2} \mathrm{~s}^{-1}$.

It is not postulated that the low-mode incoherent internal tide is the direct source of the internal wave 
continuum and mixing in the thermocline; the above discussion merely illustrates that the apparent rate of energy loss due to time-variable refraction is a significant fraction of the diabatic mixing hypothesized to maintain the thermocline. Insofar as the incoherent internal tide propagates without conversion to smallerscale internal waves, the dissipation rate will be overestimated if it is inferred simply from the convergence of the coherent baroclinic energy flux (e.g., Tian et al. 2006).

The ensemble of tidal simulations highlights the significance of interactions between the tidal and subtidal flow in the ocean, but the completeness and realism of the results is limited by several factors including both the tidal model and the SODA background fields. For example, Carter et al. (2008) found that increasing the resolution from 4 to $1 \mathrm{~km}$ in a model of Kauai Channel led to $40 \%$ increases in baroclinic generation. Modeling the entire Hawaiian Ridge at $1-\mathrm{km}$ resolution is not computationally feasible, at present, and bathymetric maps are insufficient to provide realistic topography at this scale in any case (Marks and Smith 2006). An additional factor missing from the model is the nonlinear interaction of multiple tidal frequencies, which probably contributes to the removal of energy from the coherent and incoherent tide. For example, the spring-neap cycle of the combined $M_{2}$ and $S_{2}$ tides is correlated with tidal and subtidal internal wave kinetic energy, shear, and strain (Martin et al. 2006). Proper representation of these processes likely requires higher vertical resolution and much longer integration times to reliably capture spring-neap variability. Recent efforts such as Arbic et al. (2010) are attempting to model the more complete set of physics on a global scale.

The main deficiencies of the subtidal background fields are evident in Figs. 5-7, where it is clear that SODA represents the time-varying ocean only at scales greater than $1000 \mathrm{~km}$. Because the variance of SODA steric height $(0 / 2000 \mathrm{~m})$ is only about $65 \%$ of observed along-track SSH variance, if the above-described tidal variability is a linear function of SSH variance, then it may be $35 \%$ too low. Because background currents are proportional to the gradient of the steric height, the Doppler shifting is underestimated by a larger amount, which is particularly important for the slower, higher than mode-1, internal tides. Nonetheless, by directly propagating the internal tides through an ensemble of spatially variable background fields, the results generalize previous ray-tracing studies (Park and Watts 2006; Rainville and Pinkel 2006b; Chavanne et al. 2010b) without the need to neglect specific classes of wave/mean flow interactions.

\section{Summary}

By constructing an ensemble of tidal solutions around the Hawaiian Ridge, each based on a different realization of the subtidal background flow, the coherent and incoherent $M_{2}$ tides have been estimated. Because the background fields are comprised of a filtered version of the oceanic eddy field, the present results should be regarded as a lower limit on the amount of incoherent tidal variance in the vicinity of the Hawaiian Ridge. It is found that the incoherent tide results primarily from perturbations to the phase speed of the baroclinic tide, rather than generation site processes. Both the amplitude of the incoherent tide and its energy flux are found to be spatially inhomogeneous. At a distance of $250 \mathrm{~km}$ from the ridge axis about $20 \%$ of the baroclinic energy flux is carried by the incoherent tide.

A comparison of modeled and observed SSH shows the strengths and limitations of the present approach. The wavenumber spectrum of along-track satellite SSH has more energy at higher wavenumbers than the SODAderived background fields, consistent with the filtering operations used to construct the SODA ocean-state estimates. In contrast, comparison of the along-track spectrum of harmonically analyzed SSH shows qualitative agreement with the coherent tide predicted from the ensemble.

Observed and modeled baroclinic energy flux have been compared to published data at several sites. Quantitative agreement between modeled and observed flux is not obtained and may be a consequence of bottom topography error or deficiencies in the background stratification. Because the energy flux at a point is the superposition of energy fluxes originating at multiple sites, correctly modeling the flux requires simulating both the conditions at the generation sites and the propagation from the generation to the observation sites. Our results highlight the irreducible complexity of the baroclinic tidal fields, which arises from the geometry of spatially distributed sources.

If the rate of energy conversion of the coherent-toincoherent tide is identified with the small-scale vertical buoyancy flux, then a turbulence diffusivity greater than the canonical value of $K_{V}=10^{-4} \mathrm{~m}^{2} \mathrm{~s}^{-1}$ would be inferred at a few deep-ocean sites near Hawaii, for example, near the Musicians Seamounts. To equate the adiabatic scattering (associated with time-variable refraction) with diabatic mixing at small scales requires an assumption that the incoherent baroclinic tide rapidly enters the internal wave continuum, a hypothesis which the present results do not address. Such a study would require very high vertical and horizontal resolution to accurately represent the nonlinear interactions of baroclinic waves. Nonetheless, our results demonstrate 
the significance of the incoherent tide to tidal energy budgets.

Acknowledgments. Support for this work was provided by NSF Awards OCE-9819518 (Hawaii Ocean Mixing Experiment) and OCE-0623540 (Regional Studies of the Internal Tides). The ensemble of tidal solutions was computed using resources provided by a National Center for Atmospheric Research Large-Resource Allocation. Satellite altimeter data used were extracted from the Radar Altimeter Database System (RADS; http://rads.tudelft.nl/rads/rads.shtml).

\section{APPENDIX}

\section{Dispersion Relation for Waves Propagating in a Nonuniform Medium}

The dispersion relation for inertial gravity waves propagating through nonuniform background currents has been derived by several authors (Kunze 1985; Jones 2005). A special case is derived here to justify the use of nondimensional measures of the background flow field (e.g., Rossby number and Froude number) to assess the relative importance of various processes in scattering the internal tide.

A dispersion relation may be derived by using an expansion in flat-bottom vertical modes (Hendershott 1981), which neglects modal coupling (Griffiths and Grimshaw 2007) and vertical dependence of the background fields. With these approximations, the subtidal background velocity $(\bar{u}, \bar{v})$ influences the waves through the vertical component of relative vorticity $\bar{\zeta}=\bar{v}_{x}-\bar{u}_{y}$ and through the modal eigenvalue $c_{0}^{-2}=(g \bar{h})^{-1}$ expressed in terms of the effective depth $D$.

Small-amplitude mode- 1 waves are governed by

$$
\begin{aligned}
\mathbf{u}_{t}+\overline{\mathbf{u}} \cdot \nabla \mathbf{u}+\mathbf{u} \cdot \nabla \overline{\mathbf{u}}+f \hat{k} \times \mathbf{u}+g \nabla \eta & =0 \quad \text { and } \\
\eta_{t}+D \nabla \cdot \mathbf{u} & =0,
\end{aligned}
$$

where $\mathbf{u}=(u, v)$ is the mode- 1 velocity, $\eta$ is the mode- 1 amplitude, and $f$ is the vertical component of the Coriolis term. Assuming the solution $(\mathbf{u}, \eta)=(\hat{\mathbf{u}}, \hat{\eta})$ $\exp [i(-\omega t+k x+l y)]$, propagating waves satisfy

$$
\left|\begin{array}{ccc}
-i \hat{\omega}+\bar{u}_{x} & -\left(f-\bar{u}_{y}\right) & g i k \\
+\left(f+\bar{v}_{x}\right) & -i \hat{\omega}+\bar{v}_{y} & g i l \\
\text { Dik } & \text { Dil } & -i \omega
\end{array}\right|=0
$$

where $\hat{\omega}=\omega-\mathbf{k} \cdot \overline{\mathbf{u}}$. An effective Coriolis parameter $f_{e}$ may be defined as

$$
\begin{aligned}
f_{e}^{2} & =f^{2}+f\left(\bar{v}_{x}-\bar{u}_{y}\right)+\bar{u}_{x} \bar{v}_{y}-\bar{u}_{y} \bar{v}_{x} \\
& =f^{2}+f \bar{\zeta}+s^{2},
\end{aligned}
$$

where $s^{2}=\bar{u}_{x} \bar{v}_{y}-\bar{u}_{y} \bar{v}_{x}$ is the determinant of the rate of strain matrix. The dispersion relation is then given by

$$
\begin{aligned}
& \omega\left(\hat{\omega}^{2}-f_{e}^{2}-\frac{\hat{\omega}}{\omega} g D|\mathbf{k}|^{2}\right) \\
& \quad-i\left[\left(\bar{v}_{x}+\bar{u}_{y}\right) k l-\bar{u}_{x} l^{2}-\bar{v}_{y} k^{2}\right] g D=0,
\end{aligned}
$$

and propagating internal waves correspond to roots of

$$
\hat{\omega}^{2}-f_{e}^{2}-\frac{\hat{\omega}}{\omega} g D|\mathbf{k}|^{2}=0
$$

The Doppler-shifted phase speed $c_{p}=\hat{\omega} /|\mathbf{k}|$ is thus

$$
c_{p}^{2}=g D \frac{\hat{\omega}}{\omega}+\frac{f_{e}^{2}}{|\mathbf{k}|^{2}} \quad \text { or } \quad c_{p}^{2}=\frac{g D \frac{\hat{\omega}}{\omega}}{1-\frac{f_{e}^{2}}{\hat{\omega}^{2}}}
$$

The latter result illustrates the finding in Kunze (1985) that one effect of the background flow is to rotate the reference frame in which waves propagate, leading to a change in the effective Coriolis parameter

$$
f_{e}=f\left(1+\frac{\bar{\zeta}}{f}+\frac{s^{2}}{f^{2}}\right)^{1 / 2}
$$

or

$$
f_{e} \approx f\left(1+\frac{1}{2} \mathrm{Ro}\right)
$$

where $\operatorname{Ro}=\bar{\zeta} / f \ll 1$ and $(s / f)^{2}=O\left(\mathrm{Ro}^{2}\right)$ is assumed.

One may assess the relative contributions of changing mesoscale stratification (changes in $g D$ ), relative vorticity, and Doppler shifting by taking the differential of the dispersion relation [Eq. (A6)]. Consider the component in the direction of the background flow

$$
\delta\left(c_{p}-\overline{\mathbf{u}}\right)^{2} \approx \delta(g D)+\delta f_{e}^{2} \frac{c_{p}}{\omega^{2}},
$$

where terms involving the displacement parameter $d=$ $|\overline{\mathbf{u}}||\mathbf{k}| / \omega$ have been neglected, and where $\delta$ indicates a small change in its argument. Expanding, one finds that

$$
\frac{\delta c_{p}}{c_{p}} \approx \frac{1}{2} \frac{\delta(g D)}{g D}+\frac{\delta|\overline{\mathbf{u}}|}{c_{p}}+\frac{1}{2} \frac{f^{2}}{\omega^{2}} \frac{\delta \bar{\zeta}}{f},
$$


where higher-order products with the Froude number $|\overline{\mathbf{u}}| / c_{p}$ have been neglected. Perturbations in the phase speed occur due to vertical mode eigenvalue, the Doppler shift, and relative vorticity.

The above discussion emphasizes the real part of the dispersion relation. The imaginary term in Eq. (A6) is associated with energy exchange between the waves and background flow. If the background flow is nondivergent (e.g., geostrophic) then the imaginary term takes a particularly simple form, namely,

$$
\frac{1}{2} s\left(\omega^{2}-f_{e}^{2}\right) \cos 2(\phi-\theta),
$$

where $\phi=1 / 2 \tan ^{-1}(b / a)$ is the inclination angle of the principle axis of the rate-of-strain matrix, defined by rates of stretching and shearing, $a=\bar{u}_{x}-\bar{v}_{y}$ and $b=$ $\bar{v}_{x}+\bar{u}_{y}$, respectively, $s^{2}=a^{2}+b^{2}$, and $\theta$ is the direction of the internal tide wavenumber.

\section{REFERENCES}

Alford, M., J. A. MacKinnon, Z. Zhao, R. Pinkel, J. Klymak, and T. Peacock, 2007: Internal waves across the Pacific. Geophys. Res. Lett., 34, L24601, doi:10.1029/2007GL031566.

Arbic, B. K., A. J. Wallcraft, and E. J. Metzger, 2010: Concurrent simulation of the eddying general circulation and tides in a global ocean model. Ocean Modell., 32, 175-187.

Bennett, A. F., 2002: Inverse Modeling of the Ocean and Atmosphere. Cambridge University Press, $234 \mathrm{pp}$.

Carter, G. S., and M. C. Gregg, 2006: Persistent near-diurnal internal waves observed above a site of $M_{2}$ barotropic-tobaroclinic conversion. J. Phys. Oceanogr., 36, 1136-1147.

- , and Coauthors, 2008: Energetics of $M_{2}$ barotropic to baroclinic tidal conversion at the Hawaiian Islands. J. Phys. Oceanogr., 38, 2205-2223.

Carton, J. A., and B. S. Giese, 2008: A reanalysis of ocean climate using Simple Ocean Data Assimilation (SODA). Mon. Wea. Rev., 136, 2999-3017.

- G. Chepurin, X. Cao, and B. Giese, 2000: A simple ocean data assimilation analysis of the global upper ocean. Part I: Methodology. J. Phys. Oceanogr., 30, 294-309.

Chavanne, C., P. Flament, G. Carter, M. Merrifield, D. Luther, E. D. Zaron, and K. Gurgel, 2010a: The surface expression of semidiurnal internal tides near a strong source at Hawaii. Part I: Observations and numerical predictions. J. Phys. Oceanogr., 40, 1155-1179.

, — - D. Luther, and K. Gurgel, 2010b: The surface expression of semidiurnal internal tides near a strong source at Hawaii. Part II: Interactions with mesoscale currents. J. Phys. Oceanogr., 40, 1180-1200.

Chelton, D. B., and M. G. Schlax, 1996: Global observations of oceanic Rossby waves. Science, 272, 234-238.

Cole, S. T., D. L. Rudnick, B. A. Hodges, and J. P. Martin, 2009: Observations of tidal internal wave beams at Kauai Channel, Hawaii. J. Phys. Oceanogr., 39, 421-436.

Cummins, P. F., and L.-Y. Oey, 1997: Simulations of barotropic and baroclinic tides off northern British Columbia. J. Phys. Oceanogr., 27, 762-781.
DeSzoeke, R. A., 2004: An effect of the thermobaric nonlinearity of the equation of state: A mechanism for sustaining solitary Rossby waves. J. Phys. Oceanogr., 34, 2042-2056.

Dushaw, B. D., 2002: Mapping low-mode internal tides near Hawaii using TOPEX/Poseidon altimeter data. Geophys. Res. Lett., 29 (8), doi:10.1029/2001GL013944.

—, P. F. Worcester, B. D. Cornuelle, B. M. Howe, and D. S. Luther, 1995: Baroclinic and barotropic tides in the central North Pacific Ocean determined from long-range reciprocal acoustic transmissions. J. Phys. Oceanogr., 25, 631-647.

- - , and M. A. Dzieciuch, 2011: On the predictability of mode-1 internal tides. Deep-Sea Res., 58, 677-698.

Egbert, G. D., and R. D. Ray, 2001: Estimates of $M_{2}$ tidal energy dissipation from TOPEX/Poseidon altimeter data. J. Geophys. Res., 106 (C10), 22 475-22502.

—- and S. Y. Erofeeva, 2002: Efficient inverse modeling of barotropic ocean tides. J. Atmos. Oceanic Technol., 19, 183-204.

Foreman, M. G., J. Y. Cherniawsky, and V. A. Ballantyne, 2009: Versatile harmonic tidal analysis: Improvements and applications. J. Atmos. Oceanic Technol., 26, 806-817.

Fu, L., and R. Ferrari, 2008: Observing oceanic submesoscale processes from space. Eos, Trans. Amer. Geophys. Union, 89 (48), 488-489.

Garrett, C., and E. Kunze, 2007: Internal tide generation in the deep ocean. Annu. Rev. Fluid Mech., 39, 57-87, doi:10.1146/ annurev.fluid.39.050905.110227.

Griffiths, S. D., and R. H. Grimshaw, 2007: Internal tide generation at the continental shelf modeled using a modal decomposition: Two-dimensional results. J. Phys. Oceanogr., 37, 428-451.

Hendershott, M. C., 1981: Long waves and ocean tides. Evolution of Physical Oceanography, B. A. Warren and C. Wunsch, Eds., MIT Press, 293-341.

Johnston, S. T., M. A. Merrifield, and P. E. Holloway, 2003: Internal tide scattering at the Line Islands Ridge. J. Geophys. Res., 108, 3365, doi:10.1029/2003JC001844.

Jones, R. M., 2005: A general dispersion relation for internal gravity waves in the atmosphere or ocean, including baroclinicity, vorticity, and rate of strain. J. Geophys. Res., 110, D22106, doi:10.1029/2004JD005654

Kelly, S. M., J. D. Nash, and E. Kunze, 2010: Internal-tide energy over topography. J. Geophys. Res., 115, C06014, doi:10.1029/ 2009JC005618

Klymak, J. M., and Coauthors, 2006: An estimate of tidal energy lost to turbulence at the Hawaiian Ridge. J. Phys. Oceanogr., 36, 1148-1164.

, R. Pinkel, and L. Rainville, 2008: Direct breaking of the internal tide near topography: Kaena Ridge, Hawaii. J. Phys Oceanogr., 38, 380-399.

Kunze, E., 1985: Near-inertial wave propagation in geostrophic shear. J. Phys. Oceanogr., 15, 544-565.

Large, W. G., J. C. McWilliams, and S. C. Doney, 1994: Oceanic vertical mixing: A review and a model with a nonlocal boundary layer parameterization. Rev. Geophys., 32, 363 403.

Lee, C. M., E. Kunze, T. B. Sanford, J. D. Nash, M. A. Merrifield, and P. E. Holloway, 2006: Internal tides and turbulence along the 3000-m isobath of the Hawaiian Ridge with model comparisons. J. Phys. Oceanogr., 36, 1165-1183.

Legg, S., and J. Klymak, 2008: Internal hydraulic jumps and overturning generated by tidal flow over a tall steep ridge. J. Phys. Oceanogr., 38, 1949-1964. 
Levine, M. D., and T. J. Boyd, 2006: Tidally-forced internal waves and overturns observed on a slope: Results from the HOME survey component. J. Phys. Oceanogr., 36, 1184-1201.

Lilly, D. K., 1967: The representation of small-scale turbulence in numerical simulation experiments. NCAR Manuscript 281, 24 pp.

MacKinnon, J. A., and K. B. Winters, 2005: Subtropical catastrophe: Significant loss of low-mode tidal energy at $28.9^{\circ}$. Geophys. Res. Lett., 32, L15605, doi:10.1029/2005GL023376.

Marks, K. M., and W. F. Smith, 2006: An evaluation of publicly available global bathymetry grids. Mar. Geophys. Res., 27, 19-34, doi:10.1007/s11001-00502095-4.

Martin, J. P., D. L. Rudnick, and R. Pinkel, 2006: Spatially broad observations of internal waves in the upper ocean at the Hawaiian Ridge. J. Phys. Oceanogr., 36, 1085-1103.

McCune, D., and C. Ludescher-Furth, cited 2011: PSPLINEPrinceton spline and hermite cubic interpolation routines. National Transport Code Collaboration. [Available online at http://w3.pppl.gov/ntcc/PSPLINE/.]

Middleton, D., 1960: An Introduction to Statistical Communication Theory. McGraw Hill, 1140 pp.

Muller, C., and O. Bühler, 2009: Saturation of the internal tides and induced mixing in the abyssal ocean. J. Phys. Oceanogr., 39, 2077-2096.

Nash, J. D., E. Kunze, C. M. Lee, and T. B. Sanford, 2006: Structure of the baroclinic tide generated at Kaena Ridge, Hawaii. J. Phys. Oceanogr., 36, 1123-1135.

Osborn, T. R., 1980: Estimates of the local rate of vertical diffusion from temperature measurements. J. Phys. Oceanogr., 10, 83-89.

Park, J., and D. R. Watts, 2006: Internal tides in the southwestern Japan/East Sea. J. Phys. Oceanogr., 36, 22-34.

Polzin, K. L., J. M. Toole, J. R. Ledwell, and R. W. Schmitt, 1997: Spatial variability of turbulent mixing in the abyssal ocean. Science, 276, 93-96.

Qiu, B., 1999: Seasonal eddy field modulation of the North Pacific Subtropical Countercurrent: TOPEX/Poseidon observations and theory. J. Phys. Oceanogr., 29, 2471-2486.

Rainville, L., and R. Pinkel, 2006a: Baroclinic energy flux at the Hawaiian Ridge: Observations from the R/P Flip. J. Phys. Oceanogr., 34, 1123-1135.

$\longrightarrow$, and - 2006b: Propagation of low-mode internal waves through the ocean. J. Phys. Oceanogr., 36, 1220-1236.

— T. S. Johnston, G. S. Carter, M. A. Merrifield, R. Pinkel, P. F. Worcester, and B. D. Dushaw, 2010: Interference pattern and propagation of the $M_{2}$ internal tide south of the Hawaiian Ridge. J. Phys. Oceanogr., 40, 311-325.

Ray, R. D., and G. T. Mitchum, 1996: Surface manifestation of internal tides generated near Hawaii. Geophys. Res. Lett., 23, 2101-2104.

—, and E. D. Zaron, 2011: Non-stationary internal tides observed with satellite altimetry. Geophys. Res. Lett., 38, L17609, doi:10.1029/2011GL048617.
Rea, D. K., and F. P. Naugler, 1971: Musicians Seamount province and related crustal structures north of the Hawaiian Ridge. Mar. Geol., 10, 89-111.

Schaeffer, P., Y. Faugere, J. F. Legeais, and N. Picot, cited 2011: The CNES CLS 2011 global mean sea surface. [Available online at http://www.aviso.oceanobs.com/fileadmin/documents/ OSTST/2011/oral/02_Thursday/Splinter\%202\%20GEO/02\% 20Schaeffer\%20Pres_OSTST2011_MSS_CNES_CLS2011.pdf.]

Simmons, H. L., R. W. Hallberg, and B. K. Arbic, 2004: Internal wave generation in a global baroclinic tide model. Deep-Sea Res. II, 51, 3043-3068.

Smith, W. H., and D. T. Sandwell, 1997: Global sea floor topography from satellite altimetry and ship depth soundings. Science, 277, 1956-1962.

St. Laurent, L., and C. Garrett, 2002: The role of internal tides in mixing the deep ocean. J. Phys. Oceanogr., 32, 2882-2899.

Tian, J., L. Zhou, and X. Zhang, 2006: Latitudinal distribution of mixing rate caused by $M_{2}$ internal tide. J. Phys. Oceanogr., 36, $35-42$.

Wunsch, C., 1975: Internal tides in the ocean. Rev. Geophys., 13, 167-182.

— , and R. Ferrari, 2004: Vertical mixing, energy, and the general circulation of the oceans. Annu. Rev. Fluid Mech., 36, 281-314, doi:10.1146/annurev.fluid.36.050802.122121.

Zaron, E. D., and G. D. Egbert, 2006a: Estimating open-ocean barotropic tidal dissipation: The Hawaiian Ridge. J. Phys. Oceanogr., 36, 1019-1035.

$\longrightarrow$, and - , 2006b: Verification studies for a $z$-coordinate primitive-equation model: Tidal conversion at a midocean ridge. Ocean Modell., 14, 257-278, doi:10.1016/ j.ocemod.2006.05.007.

— and - 2007: The impact of the $M_{2}$ internal tide on dataassimilative model estimates of the surface tide. Ocean Modell., 18, 210-216, doi:10.1016/j.ocemod.2007.05.001.

_ C. Chavanne, G. D. Egbert, and P. Flament, 2009: Baroclinic tidal generation in the Kauai Channel inferred from HF-Radar. Dyn. Atmos. Oceans, 48, 93-120, doi:10.1016/ j.dynatmoce.2009.03.002.

Zhao, Z., and M. H. Alford, 2009: New altimetric estimates of mode- $1 M_{2}$ internal tides in the central North Pacific Ocean. J. Phys. Oceanogr., 39, 1669-1684.

,-- J. A. MacKinnon, and R. Pinkel, 2010: Long-range propagation of the semidiurnal internal tide from the Hawaiian Ridge. J. Phys. Oceanogr., 40, 713-736.

,-- J. Girton, T. M. Johnston, and G. Carter, 2011: Internal tides around the Hawaiian Ridge estimated from multisatellite altimetry. J. Geophys. Res., 116, C12039, doi:10.1029/ 2011JC007045.

Zilberman, N. V., M. A. Merrifield, G. S. Carter, D. S. Luther, M. D. Levine, and T. J. Boyd, 2011: Incoherent nature of $M_{2}$ internal tides at the Hawaiian Ridge. J. Phys. Oceanogr., 41, 3021-2036. 